Preprint typeset in JHEP style - HYPER VERSION

Imperial/TP/08/DW/01

\title{
M-theory, exceptional generalised geometry and superpotentials
}

\author{
Paulo Pires Pacheco \\ Department of Physics, Imperial College London \\ London, SW7 2AZ, UK \\ Daniel Waldram \\ Department of Physics, Imperial College London \\ London, SW7 2AZ, UK \\ Institute for Mathematical Sciences, Imperial College London \\ London, SW7 $2 A Z, U K$
}

\begin{abstract}
We discuss the structure of "exceptional generalised geometry" (EGG), an extension of Hitchin's generalised geometry that provides a unified geometrical description of backgrounds in eleven-dimensional supergravity. On a $d$-dimensional background, as first described by Hull, the action of the generalised geometrical $O(d, d)$ symmetry group is replaced in EGG by the exceptional U-duality group $E_{d(d)}$. The metric and form-field degrees of freedom combine into a single geometrical object, so that EGG naturally describes generic backgrounds with flux, and there is an EGG analogue of the Courant bracket which encodes the differential geometry. Our focus is on the case of seven-dimensional backgrounds with $N=1$ four-dimensional supersymmetry. The corresponding EGG is the generalisation of a $G_{2}$-structure manifold. We show it is characterised by an element $\phi$ in a particular orbit of the $\mathbf{9 1 2}$ representation of $E_{7(7)}$, which defines an $S U(7) \subset E_{7(7)}$ structure. As an application, we derive the generic form of the four-dimensional effective superpotential, and show that it can be written in a universal form, as a homogeneous $E_{7(7)}$-invariant functional of $\phi$.
\end{abstract}




\section{Contents}

1. Introduction 1

2. EGG in seven dimensions 3

2.1 Review of generalised geometry 3

2.2 The exceptional generalised tangent space and $E_{7(7)}$

2.3 The exceptional generalised metric and $S U(8) / \mathbb{Z}_{2}$ structures 9

3. Supersymmetric backgrounds and EGG 11

3.1 Effective theories and field decompositions 11

3.2 Review of generalized geometry of $N=2$ type II backgrounds 13

$3.3 \quad N=1$ M-theory backgrounds, EGG and $S U(7)$ structures

4. Application: the effective superpotential 18

4.1 Generic form of the effective superpotential $\overline{19}$

4.2 An $E_{7(7)}$ covariant expression 20

5. Conclusions 23

A. Conventions 25

A.1 Eleven-dimensional supergravity 25

A.2 Cliff $(7,0)$ and seven-dimensional spinors $\quad 26$

B. The exceptional Lie group $E_{7(7)}$

B.1 Definition and the $\mathbf{5 6}$ representation 27

B.2 The 133 and 912 representations 28

B.3 A $G L(7)$ subgroup 29

B.4 The $S U(8) / \mathbb{Z}_{2}$ subgroup and spinor indices 30

\section{Introduction}

Type II string backgrounds in $d$ dimensions which include non-trivial fluxes have a natural description in terms of Hitchin's generalised geometry [1, 2, 3, , 4, where the metric and NS-NS $B$-field are combined into a single geometrical object, transforming under $O(d, d)$. This description has proved very useful in, among other things: characterising supersymmetric backgrounds, finding new examples with non-zero fluxes and writing supersymmetric low-energy effective theories [5]-25]; describing topological string theories and generic $N=(2,2) \sigma$-models [26] 39]; as well as motivating the structure of non-geometrical backgrounds [4]]- 48]. 
The aim of this paper is to understand some details of how similar constructions based on the exceptional groups $E_{d(d)}$ can be used to describe M-theory, or more precisely, eleven-dimensional supergravity backgrounds. The general form of such constructions, as well as those arising from type II theories, has been described recently by Hull [49]. That work was partially motivated by the existence of so-called "non-geometrical" backgrounds, which appear consistent as string theory vacua and are typically dual to supergravity backgrounds, but do not themselves have a consistent global description in supergravity. This suggested considering extensions of generalised geometry based on generic $O(d, d)$ or $E_{d(d)}$ bundles, whereas only a subclass of such bundles arise in supergravity. The corresponding geometry was generically dubbed "extended" (or more specifically "M-geometry" for the generalisation of eleven-dimensional supergravity).

Here, however, we will consider only supergravity backgrounds and concentrate on the physically important example of seven-dimensional backgrounds and hence the group $E_{7(7)}$. There are two parts to the analysis. First to build the analog of the generalised geometry and then to describe the geometrical objects that characterise supersymmetric $N=1$ backgrounds in four dimensions. In analogy to the type II analysis in [11, 20], we consider the particular application of writing the $N=1$ superpotential in a generic $E_{7(7)}$-invariant form.

The essential idea of the construction is that $O(d, d)$ symmetries of generalised $d$ dimensional geometry, which in string theory are related to T-duality symmetries, should be replaced by the U-duality exceptional symmetry groups $E_{d(d)}$ [50, 51, 52]. Since the Uduality connects all the bosonic degrees of freedom of eleven-dimensional supergravity, or for type II theories, both NS-NS and R-R degrees of freedom, this extension should provide a geometrisation of generic flux backgrounds. The fact that the full eleven-dimensional supergravity could be reformulated in terms of $E_{d(d)}$ objects was first pointed out by de Wit and Nicolai [53] (for $E_{7(7)}$ ) and then by Nicolai [54] and Koepsell, Nicolai and Samtleben [55] (for $\left.E_{8(8)}\right)$, the latter calling the construction "exceptional geometry". Motivated by these authors' and Hull's nomenclature 449, we will refer to the variant of Hitchin's generalised geometry relevant to eleven-dimensional (and type II) supergravity as EGG for "exceptional generalised geometry". This reserves "extended" and " $\mathrm{M}$ " for generic M-theory backgrounds, potentially including non-geometrical examples.

We should note here that recently there have also been more ambitious related proposals connecting infinite-dimensional exceptional algebras to supergravity. The original proposal of [56, 57] had the goal of giving an eleven-dimensional covariant formulation of M-theory with $E_{11}$ invariance, as well as of lower-dimensional gauged supergravities [58], while the work of [59] describes a gauged-fixed version of the supergravity dynamics in terms of an explicit $E_{10}$ coset construction.

The paper is arranged as follows. In section 2, after briefly reviewing generalised geometry, we describe how the analogous $E_{7(7)}$-invariant EGG can be defined for a sevendimensional manifold $M$. Much of this analysis appears in 499. The corresponding exceptional generalised tangent space (EGT) encodes all the topological information of the conventional tangent space $T M$ as well as the topology of the "gerbe", the analogue of a $U(1)$-bundle, on which the supergravity three-form $A$ is a connection. We give new details 
on how the tangent space bundle embeds into the EGG, as well as the precise form of the gerbes. We also define the analogue of the Courant bracket on the EGT which encodes the differential geometry. We then introduce the notion of an $S U(8) / \mathbb{Z}_{2}$ structure on the EGT and show that this encodes supergravity metric $g$ and three-form $A$. (This is equivalent to the result [49], familiar from toroidal reductions [50], that they parameterize an element of the coset space $E_{7(7)} /\left(S U(8) / \mathbb{Z}_{2}\right)$.) We show in particular how the non-linear Chern-Simons-like terms for $A$ appear naturally in this formalism.

In section 3, we again start with review. We discuss the pure $O(6,6)$ spinors $\Phi^{ \pm}$ which characterise generalised geometrical six-dimensional type II backgrounds preserving $N=2$ supersymmetry in four dimensions, and define an $S U(3) \times S U(3)$ structure on the generalised tangent space. We then make the analogous analysis for EGG. (A short related discussion of supersymmetric backgrounds appeared in section 8 of [49].) Without flux, for the background to have $N=1$ supersymmetry in four dimensions, the compactification manifold must have a $G_{2}$-structure. We show that the generalisation to EGG is that the EGT must admit an $S U(7)$ structure. This is equivalent to the existence of a nowhere vanishing tensor $\phi$ transforming in the $\mathbf{9 1 2}$ of $E_{7(7)}$. It is not a generic element but, to be stabilized by $S U(7)$ must lie in a particular orbit under $E_{7(7)}$.

In section 1 we give an application of the these results. We first derive the form of the superpotential for a generic $N=1$ compactification of eleven-dimensional supergravity slightly extending the results of [60]. We then use the structure $\phi$, which plays the role of the chiral scalar fields in the four-dimensional theory, to show that the superpotential can be rewritten in a $E_{7(7)}$-invariant form.

We conclude in section 5 with a brief discussion of our results and of possible further work.

\section{EGG in seven dimensions}

In this section we discuss the structure of exceptional generalised geometry, focusing on the case of seven dimensions. Much of this analysis also appears in 49. Here we approach the construction from the point of view of geometrical structures and include some new details on the gerbe topology and tangent space embedding, as well the definition of the analogue of the Courant bracket. Our particular motivation will be compactification of eleven-dimensional supergravity to a four-dimensional effective theory. However here we

will first simply introduce the EGG formalism and leave the more detailed connection to supergravity to the following section. We start by reviewing the structure of generalised geometry [1, 2, 3, 4] since the EGG structure arises in a very analogous way.

\subsection{Review of generalised geometry}

In defining generalised geometry [1, 2, 3, 4, on a $d$-dimensional manifold, one starts with the (untwisted) generalised tangent space $E_{0}=T M \oplus T M^{*}$. Let us denote elements of $E_{0}$ by $X=x+\xi$. There is then a natural $O(d, d)$-invariant metric $\eta$ on $E$ given by

$$
\eta(X, X):=i_{x} \xi
$$


In particular, $\eta$ is invariant under the $G L(d) \subset O(d, d)$ action on the fibres of $T M$ and $T^{*} M$. The metric is also invariant under so called " $B$-shifts" where for any $B \in \Lambda^{2} T^{*} M$ we define

$$
\mathrm{e}^{B} X:=x+\left(\xi-i_{x} B\right) .
$$

The differential geometry of the generalised tangent space is encoded in the Courant bracket which generalises the Lie bracket between two vectors. It is defined by

$$
[x+\xi, y+\zeta]=[x, y]+\mathcal{L}_{x} \zeta-\mathcal{L}_{y} \xi-\frac{1}{2} \mathrm{~d}\left(i_{x} \zeta-i_{y} \xi\right)
$$

where $[x, y]$ is the usual Lie bracket of vector fields and $\mathcal{L}_{x}$ is the Lie derivative, so $\mathcal{L}_{x} \alpha=$ $i_{x} \mathrm{~d} \alpha+\mathrm{d} i_{x} \alpha$ for any form $\alpha$. Note that its automorphism group is not the full group of local $O(d, d)$ transformations but only the subgroup generated by diffeomorphisms and the $B$-shifts (2.2) (with $\mathrm{d} B=0$ ). Formally we can write this as the semidirect product $\operatorname{Diff}(M) \ltimes \Omega_{\text {closed }}^{2}(M)$.

The usefulness of generalised geometry in string theory and supergravity is that the ordinary metric $g$ and NS-NS two-form field $B$ combine naturally into a single object, the so-called generalised metric $G$ [2]. This is an $O(2 d)$-invariant metric which is compatible with $\eta$, that is $\eta^{-1} G \eta^{-1}=G^{-1}$. If we split into $T M$ and $T^{*} M$, we can write the $O(d, d)$ metric as the matrix

$$
\eta=\frac{1}{2}\left(\begin{array}{ll}
0 & \mathbb{1} \\
\mathbb{1} & 0
\end{array}\right), \quad \text { where } \quad X=\left(\begin{array}{l}
x \\
\xi
\end{array}\right) .
$$

The generic generalised metric can then be written as

$$
G=\frac{1}{2}\left(\begin{array}{cc}
g-B g^{-1} B & B g^{-1} \\
-g^{-1} B & g^{-1}
\end{array}\right)
$$

where $g$ is an ordinary Riemannian metric, and $B \in \Lambda^{2} T^{*} M$ is the NS-NS two-form.

Note that one can also define $G$ as a product structure $\Pi$. Writing $\Pi=\eta^{-1} G$ one has $\Pi^{2}=\mathbb{1}$, and in addition $\eta(X, Y)=\eta(\Pi X, \Pi Y)$. Hence $\Pi$ is a product structure, compatible with $\eta$, and the projections $\frac{1}{2}(\mathbb{1} \pm \Pi)$ project onto two $d$-dimensional subspaces $C^{ \pm}$, such that $E=C^{+} \oplus C^{-}$. From this perspective, given $\eta$, one sees that $G$ defines an $O(d) \times O(d)$ structure, and $\eta$ and $G$ decompose into the separate metrics on $C^{+}$and $C^{-}$. One can view $g$ and $B$ as parametrising the coset space $O(d, d) / O(d) \times O(d)$.

One can also write

$$
G(X, Y)=G_{0}\left(\mathrm{e}^{B} X, \mathrm{e}^{B} Y\right)
$$

where

$$
G_{0}=\frac{1}{2}\left(\begin{array}{cc}
g & 0 \\
0 & g^{-1}
\end{array}\right)
$$

If $B$ leads to a non-trivial flux $H$ it is only locally defined as a two-form. Globally one must patch by gauge transformations, so on the overlap $U_{(\alpha)} \cap U_{(\beta)}$ one has

$$
B_{(\alpha)}-B_{(\beta)}=\mathrm{d} \Lambda_{(\alpha \beta)},
$$


(so $\left.\Lambda_{(\alpha \beta)}=-\Lambda_{(\beta \alpha)}\right)$ while on the triple overlap $U_{(\alpha)} \cap U_{(\beta)} \cap U_{(\gamma)}$

$$
\Lambda_{(\alpha \beta)}+\Lambda_{(\beta \gamma)}+\Lambda_{(\gamma \alpha)}=\mathrm{d} \Lambda_{(\alpha \beta \gamma)},
$$

(implying $\Lambda_{(\alpha \beta \gamma)}=-\Lambda_{(\alpha \gamma \beta)}$ etc.). Mathematically this means $B$ is a connection on a gerbe (see for instance [61]). If the flux is quantised $H \in H^{3}(M, \mathbb{Z})$ then one has $g_{(\alpha \beta \gamma)}=$ $\mathrm{e}^{\mathrm{i} \Lambda_{(\alpha \beta \gamma)}} \in U(1)$ and these elements satisfy a cocycle condition on $U_{(\alpha)} \cap U_{(\beta)} \cap U_{(\gamma)} \cap U_{(\delta)}$

$$
g_{(\beta \gamma \delta)} g_{(\alpha \gamma \delta)}^{-1} g_{(\alpha \beta \delta)} g_{(\alpha \beta \gamma)}^{-1}=1 .
$$

Formally the $g_{(\alpha \beta \gamma)}$ define the gerbe, while the $\Lambda_{(\alpha \beta)}$ define a "connective structure" on the gerbe. Together they encode the analogue of the topological data of a $U(1)$ gauge bundle.

If $B$ is non-trivial, the form of the generalised metric (2.6) means that $G$ cannot really be an inner product on sections of $E_{0}=T M \oplus T^{*} M$. Instead, the generalised vectors must be sections of an extension $E$

$$
0 \longrightarrow T^{*} M \longrightarrow E \longrightarrow T M \longrightarrow 0
$$

where on the intersection of two patches $U_{\alpha} \cap U_{\beta}$ one identifies $X_{(\alpha)}=\mathrm{e}^{-\mathrm{d} \Lambda_{(\alpha \beta)}} X_{(\beta)}$, or in components

$$
x_{(\alpha)}+\xi_{(\alpha)}=x_{(\beta)}+\left(\xi_{(\beta)}+i_{x_{(\beta)}} \mathrm{d} \Lambda_{(\alpha \beta)}\right) .
$$

Here $\Lambda_{(\alpha \beta)}$ is the same one-form that appears in defining the connection $B$ (though of course it is independent of the particular choice of $B$ ). Thus we see that $E$ encodes both the topological structure of the tangent space $T M$ and the connective structure of the gerbe. Note that the form of the twisting (2.12) is such that, since the Courant bracket is $B$-shift invariant (when $B$ is closed), it can still be defined on sections of the twisted $E$.

\subsection{The exceptional generalised tangent space and $E_{7(7)}$}

We would now like to describe an exceptional generalised geometry (EGG), analogous to the generalised geometry of Hitchin, but relevant to the description of eleven-dimensional supergravity rather than simply the NS-NS sector of type II. We will concentrate on the case of a seven-dimensional manifold $M$. The basic construction has been described, in general dimension $d$, in [49] and is closely related to the work of [53, 54, 55].

Introducing the generalised tangent space allowed one to construct objects transforming under $O(d, d)$. The $g$ and $B$ degrees of freedom, parametrising the generalised metric $G$, then define an element in the $O(d, d) / O(d) \times O(d)$ coset. This coset structure is familiar from the moduli space of toroidal compactifications of NS-NS sector ten-dimensional supergravity to $10-d$ dimensions (see for instance [62]). In addition, there is, of course, a stringy $O(d, d ; \mathbb{Z})$ T-duality symmetry relating equivalent compactifications. The string winding and momentum charges transform in the $2 d$-dimensional vector representation of $O(d, d)$, namely $T M \oplus T^{*} M$.

If one includes Ramond-Ramond fields, or equivalently considers toroidal compactifi-

cations of eleven-dimensional supergravity [50, 51], the moduli spaces are cosets $E_{d(d)} / H$ (where $E_{d(d)}$ is the maximally non-compact real form of the exceptional group and $H$ is 
the corresponding maximal compact subgroup). Elements in the coset are parametrised by the components of the eleven-dimensional supergravity fields on the compact space, namely the metric $g$ and three-form $A$ potential (and potentially its dual six-form $\tilde{A}$ ). The discrete subgroup $E_{(d(d)}(\mathbb{Z})$ is the U-duality group relating different equivalent M-theory backgrounds [52]. The momentum and brane charges fill out a particular representation of $E_{d(d)}$. In particular, in $d=7$, the momentum, membrane, fivebrane and Kaluza-Klein monopole charges [63] fill out the 56 representation of $E_{7(7)}$. (Note that the definition of $E_{7(7)}$, together with some details of its various representations, is summarised in appendix B.)

Given this extension from the T-duality group $O(d, d)$ to U-duality group $E_{d(d)}$, it is natural to introduce a corresponding extension of generalised geometry. For $d=7$, the analogue of the generalised tangent space is an "exceptional generalised tangent space" (EGT) which transforms in the 56 representation of $E_{7(7)}$. As in generalised geometry, the $G L(7, \mathbb{R})$ structure group of the tangent and cotangent spaces should be a subgroup of $E_{7(7)}$, and we also expect the gauge transformations of $A$, like the $B$-shifts in generalised geometry, to be somehow embedded into $E_{7(7)}$.

The construction of the EGT is as follows. As described in appendix B.1, there is an $S L(8, \mathbb{R})$ subgroup of $E_{7(7)}$ under which the $\mathbf{5 6}$ representation is given by

$$
E_{0}=\Lambda^{2} V \oplus \Lambda^{2} V^{*}
$$

where $V$ is the eight-dimensional fundamental representation of $S L(8, \mathbb{R})$. The $G L(7, \mathbb{R})$ structure group of the tangent space $T M$ embeds as (see appendix B.3)

$$
V=\left[\left(\Lambda^{7} T^{*} M\right)^{1 / 4} \otimes T M\right] \oplus\left(\Lambda^{7} T^{*} M\right)^{-3 / 4} .
$$

One then finds

$$
E_{0}=\left(\Lambda^{7} T^{*} M\right)^{-1 / 2} \otimes\left[T M \oplus \Lambda^{2} T^{*} M \oplus \Lambda^{5} T^{*} M \oplus\left(T^{*} M \otimes \Lambda^{7} T^{*} M\right)\right]
$$

(Note that the final term in brackets can also be written as $\left.\left(\Lambda^{7} T^{*} M\right)^{2} \otimes \Lambda^{6} T M\right)$. The bundle $\left(\Lambda^{7} T^{*} M\right)^{-1 / 2}$ is isomorphic to the trivial bundle, thus there is always a (non-canonical) isomorphism

$$
E_{0} \simeq T M \oplus \Lambda^{2} T^{*} M \oplus \Lambda^{5} T^{*} M \oplus\left(T^{*} M \otimes \Lambda^{7} T^{*} M\right) .
$$

It is $E_{0}$ which is the (untwisted) exceptional generalised tangent space ${ }^{1}$. Except for the overall tensor density factor of $\left(\Lambda^{7} T^{*} M\right)^{-1 / 2}$, we see that we can identify it as a sum of vectors, two-forms, five-forms and one-forms tensor seven-forms. Given the isomorphism (2.16) we can write

$$
X=x+\omega+\sigma+\tau \in E_{0},
$$

\footnotetext{
${ }^{1}$ Note that there is a second possible way to embed $G L(7, \mathbb{R})$, and hence choice for $E_{0}$, analogous to the choice of spin-structures of $O(d, d)$ 2], where $E_{0}$ is defined as in (2.15) except with an overall factor of $\Lambda^{7} T M /\left|\Lambda^{7} T M\right|$. This bundle has a similar isomorphism to (2.16) but with $T M$ and $T^{*} M$ exchanged everywhere.
} 
where, writing the $G L(7, \mathbb{R})$ indices explicitly, we have $x^{m}, \omega_{m n}, \sigma_{m_{1} \ldots m_{5}}$ and $\tau_{m, n_{1} \ldots n_{7}}$. Physically in M-theory we expect these to correspond to momentum, membrane, fivebrane and Kaluza-Klein monopole charge respectively.

Recall that the T-duality symmetry group acting on the generalised tangent space was defined in terms of a natural $O(d, d)$-invariant metric. As discussed in appendix B.1, the group $E_{7(7)}$ is defined by, not a metric, but a symplectic structure $\Omega$ and symmetric quartic invariant $q$ on the 56-dimensional representation space. These are given explicitly in terms of $S L(8, \mathbb{R})$ representations in the appendix (B.1), and are by definition $G L(7, \mathbb{R})$, hence diffeomorphism, invariant.

Having identified the $G L(7, \mathbb{R})$ tangent space symmetry in $E_{7(7)}$, we would next like to identify the analogues of the $B$-shifts. This is essentially contained in the original dimensional reduction of eleven-dimensional supergravity on $T^{7}$ [50]. We note that the 133-dimensional adjoint representation of $E_{7(7)}$ decomposes under $G L(7, \mathbb{R})$ as

$$
A=\left(T M \otimes T^{*} M\right) \oplus \Lambda^{6} T M \oplus \Lambda^{6} T^{*} M \oplus \Lambda^{3} T M \oplus \Lambda^{3} T^{*} M .
$$

Given there is a three-form potential $A$ in eleven-dimensional supergravity, the analogue of $B$-shifts should be $A$-shifts generated by $A \in \Lambda^{3} T^{*} M$. In fact, we will also consider $\tilde{A}$-shifts with $\tilde{A} \in \Lambda^{6} T^{*} M$ corresponding to the dual six-form potential. This will be described in more detail in the next section. For now we simply note that $A$ and $\tilde{A}$ are both elements of the adjoint bundle (2.18). Their action on $X \in E$ is given in $(\overline{B .23})$. It exponentiates to

$$
\begin{aligned}
& \mathrm{e}^{A+\tilde{A}} X=x+\left[\omega+i_{x} A\right]+\left[\sigma+A \wedge \omega+\frac{1}{2} A \wedge i_{x} A+i_{x} \tilde{A}\right] \\
& \quad+\left[\tau+j A \wedge \sigma-j \tilde{A} \wedge \omega+j A \wedge i_{x} \tilde{A}+\frac{1}{2} j A \wedge A \wedge \omega+\frac{1}{6} j A \wedge A \wedge i_{x} A\right]
\end{aligned}
$$

where we are using a notion for elements of $T^{*} M \otimes\left(\Lambda^{7} T^{*} M\right)$ defined in (B.24). Note that the action truncates at cubic order. The corresponding Lie algebra, unlike the case of $B$-shifts is not Abelian. We have the commutator

$$
\left[A+\tilde{A}, A^{\prime}+\tilde{A}^{\prime}\right]=-A \wedge A^{\prime} .
$$

That is, two $A$-shifts commute to give a $\tilde{A}$ shift 64 .

When $A$ and $\tilde{A}$ are non-trivial one is led to defining a twisted EGT which encodes the patching of the potentials. This is again completely analogous to the generalised geometrical case. One starts with

$$
X_{0} \in T M \oplus \Lambda^{2} T^{*} M \oplus \Lambda^{5} T^{*} M \oplus\left(T^{*} M \otimes \Lambda^{7} T^{*} M\right)
$$

On a given patch $U_{(\alpha)}$ we define the shifted element

$$
X_{(\alpha)}=\mathrm{e}^{A_{(\alpha)}+\tilde{A}_{(\alpha)}} X_{0}
$$

In passing from one patch to another we have, on $U_{(\alpha)} \cap U_{(\beta)}$,

$$
X_{(\alpha)}=\mathrm{e}^{\mathrm{d} \Lambda_{(\alpha \beta)}+\mathrm{d} \tilde{\Lambda}_{(\alpha \beta)}} X_{(\beta)},
$$


provided the connections $A$ and $\tilde{A}$ patch as

$$
\begin{aligned}
& A_{(\alpha)}-A_{(\beta)}=\mathrm{d} \Lambda_{(\alpha \beta)}, \\
& \tilde{A}_{(\alpha)}-\tilde{A}_{(\beta)}=\mathrm{d} \tilde{\Lambda}_{(\alpha \beta)}-\frac{1}{2} \mathrm{~d} \Lambda_{(\alpha \beta)} \wedge A_{(\beta)} .
\end{aligned}
$$

As we will see in the next section this corresponds exactly to the patching of the threeand six-form potentials arising from eleven-dimensional supergravity.

The patching (2.23) imply that $X_{(\alpha)}$ are sections of a twisted EGT which we will denote as $E$. Explicitly in components we have

$$
\begin{aligned}
x_{(\alpha)}= & x_{(\beta)}, \\
\omega_{(\alpha)}= & \omega_{(\beta)}+i_{x_{(\beta)}} \mathrm{d} \Lambda_{(\alpha \beta)}, \\
\sigma_{(\alpha)}= & \sigma_{(\beta)}+\mathrm{d} \Lambda_{(\alpha \beta)} \wedge \omega_{(\beta)}+\frac{1}{2} \mathrm{~d} \Lambda_{(\alpha \beta)} \wedge i_{x_{(\beta)}} \mathrm{d} \Lambda_{(\alpha \beta)}+i_{x_{(\beta)}} \mathrm{d} \tilde{\Lambda}_{(\alpha \beta)}, \\
\tau_{(\alpha)}= & \tau_{(\beta)}+j \mathrm{~d} \Lambda_{(\alpha \beta)} \wedge \sigma_{(\beta)}-j \mathrm{~d} \tilde{\Lambda}_{(\alpha \beta)} \wedge \omega_{(\beta)}+j \mathrm{~d} \Lambda_{(\alpha \beta)} \wedge i_{x_{(\beta)}} \mathrm{d} \tilde{\Lambda}_{(\alpha \beta)} \\
& \quad+\frac{1}{2} j \mathrm{~d} \Lambda_{(\alpha \beta)} \wedge \mathrm{d} \Lambda_{(\alpha \beta)} \wedge \omega_{(\beta)}+\frac{1}{6} j \mathrm{~d} \Lambda_{(\alpha \beta)} \wedge \mathrm{d} \Lambda_{(\alpha \beta)} \wedge i_{x_{(\beta)}} \mathrm{d} \Lambda_{(\alpha \beta)}
\end{aligned}
$$

One can define $E$ formally via a series of extensions

$$
\begin{gathered}
0 \longrightarrow \Lambda^{2} T^{*} M \longrightarrow E^{\prime \prime} \longrightarrow T M \longrightarrow 0, \\
0 \longrightarrow \Lambda^{5} T^{*} M \longrightarrow E^{\prime} \longrightarrow E^{\prime \prime} \longrightarrow 0, \\
0 \longrightarrow T^{*} M \otimes \Lambda^{7} T^{*} M \longrightarrow E \longrightarrow E^{\prime} \longrightarrow 0,
\end{gathered}
$$

in analogy with 2.11).

As for the $B$-field, the potentials $A$ and $\tilde{A}$ are formally connections on gerbes. To define the connective structure of the gerbe we must define the patchings on successively higher-order intersections. For $A$, on the corresponding multiple intersections of patches we have

$$
\begin{aligned}
& \Lambda_{(\alpha \beta)}+\Lambda_{(\beta \gamma)}+\Lambda_{(\gamma \alpha)}=\mathrm{d} \Lambda_{(\alpha \beta \gamma)} \quad \text { on } U_{(\alpha)} \cap U_{(\beta)} \cap U_{(\gamma)}, \\
& \Lambda_{(\beta \gamma \delta)}-\Lambda_{(\alpha \gamma \delta)}+\Lambda_{(\alpha \beta \delta)}-\Lambda_{(\alpha \beta \gamma)}=\mathrm{d} \Lambda_{(\alpha \beta \gamma \delta)} \quad \text { on } U_{(\alpha)} \cap U_{(\beta)} \cap U_{(\gamma)} \cap U_{(\delta)} \text {. }
\end{aligned}
$$

For a quantised flux $\mathcal{F}=\mathrm{d} A_{(\alpha)}$ we have $g_{(\alpha \beta \gamma \delta)}=\mathrm{e}^{\mathrm{i} \Lambda_{(\alpha \beta \gamma \delta)}} \in U(1)$ with the cocycle condition

$$
g_{(\beta \gamma \delta \epsilon)} g_{(\alpha \gamma \delta \epsilon)}^{-1} g_{(\alpha \beta \delta \epsilon)} g_{(\alpha \beta \gamma \epsilon)}^{-1} g_{(\alpha \beta \gamma \delta)}=1,
$$

on $U_{(\alpha)} \cap \cdots \cap U_{(\epsilon)}$. For $\tilde{\Lambda}_{(\alpha \beta)}$ there is a similar set of structures, with the final cocycle condition defined on a octuple intersection $U_{\left(\alpha_{1}\right)} \cap \cdots \cap U_{\left(\alpha_{8}\right)}$.

The bundle $E$ encodes all the topological information of the supergravity background: the twisting of the tangent space $T M$ as well as the patching of the form potentials, but, as for (2.11) is independent of the particular choice of $A$ and $\tilde{A}$.

Finally we would like to identify the analogue of the Courant bracket for the EGT. We look for a pairing with $A$ - and $\tilde{A}$-shifts as automorphisms when $\mathrm{d} A=\mathrm{d} \tilde{A}=0$. One finds 
the unique "exceptional Courant bracket" (ECB):

$$
\begin{aligned}
& {\left[x+\omega+\sigma+\tau, x^{\prime}+\omega^{\prime}+\sigma^{\prime}+\tau^{\prime}\right]=} \\
& \left.\qquad x, x^{\prime}\right]+\mathcal{L}_{x} \omega^{\prime}-\mathcal{L}_{x^{\prime}} \omega-\frac{1}{2} \mathrm{~d}\left(i_{x} \omega^{\prime}-i_{x^{\prime}} \omega\right) \\
& \quad+\mathcal{L}_{x} \sigma^{\prime}-\mathcal{L}_{x^{\prime}} \sigma-\frac{1}{2} \mathrm{~d}\left(i_{x} \sigma^{\prime}-i_{x^{\prime}} \sigma\right)+\frac{1}{2} \omega \wedge \mathrm{d} \omega^{\prime}-\frac{1}{2} \omega^{\prime} \wedge \mathrm{d} \omega \\
& \quad+\frac{1}{2} \mathcal{L}_{x} \tau^{\prime}-\frac{1}{2} \mathcal{L}_{x^{\prime}} \tau+\frac{1}{2}\left(j \omega \wedge \mathrm{d} \sigma^{\prime}-j \sigma^{\prime} \wedge \mathrm{d} \omega\right)-\frac{1}{2}\left(j \omega^{\prime} \wedge \mathrm{d} \sigma-j \sigma \wedge \mathrm{d} \omega^{\prime}\right)
\end{aligned}
$$

If $G_{\text {closed }}(M)$ is the group generated by closed $A$ and $\tilde{A}$ shifts, the ECB is invariant under the $\operatorname{Diff}(M) \ltimes G_{\text {closed }}(M)$.

\subsection{The exceptional generalised metric and $S U(8) / \mathbb{Z}_{2}$ structures}

Having defined the EGT, its topology and the corresponding bracket, one can then introduce, as in [49], the analog of the generalised metric which encodes the fields of elevendimensional supergravity. The motivation is that, when compactified on $T^{7}$, the moduli

arising from the eleven-dimensional supergravity fields $g$ and $A$ parametrise a $E_{7(7)} /\left(S U(8) / \mathbb{Z}_{2}\right)$ coset space [50]. Rather than consider a fixed element of the coset space, one takes one that is a function of position in the manifold $M$ [53]. In the following, instead of starting with the coset, we show how the exceptional generalised metric can be defined as a generic $S U(8) / \mathbb{Z}_{2}$ structure on the EGT.

Fixing an element of the coset space $E_{7(7)} /\left(S U(8) / \mathbb{Z}_{2}\right)$ is equivalent to choosing a particular $S U(8) / \mathbb{Z}_{2}$ subgroup of $E_{7(7)}$. Making such a choice at each point in $M$ corresponds geometrically to a $S U(8) / \mathbb{Z}_{2}$ structure on $E$. Such a structure can be defined as follows. The $\mathbf{5 6}$ representation decomposes into $\mathbf{2 8}+\overline{\mathbf{2 8}}$ under $S U(8) / \mathbb{Z}_{2}$. Thus an $S U(8) / \mathbb{Z}_{2}$ structure is equivalent to the existence of a decomposition of the (complexified) EGT $E \otimes \mathbb{C}=C \oplus \bar{C}$ where the fibres of $C$ transform in the $\mathbf{2 8}$ of $S U(8) / \mathbb{Z}_{2}$. However this is the same as an almost complex structure $J$ with $J^{2}=-\mathbb{1}$ on $E$. For $J$ to define an $S U(8) / \mathbb{Z}_{2}$ subgroup it must also be compatible with the $E_{7(7)}$ structure. Recall that the latter was defined by a symplectic form $\Omega$ and a quartic invariant $q$. Compatibility requires

$$
\Omega(J X, J Y)=\Omega(X, Y), \quad q(J X)=q(X),
$$

or in other words $J \in E_{7(7)}$. (Note the that first condition is just the usual condition between a symplectic and an almost complex structure required to define an Hermitian metric.) Such an almost complex structure $J$ defines an $S U(8) / \mathbb{Z}_{2}$ structure on $E$.

In contrast to the generalised geometry case where the $O(d) \times O(d)$ structure was equivalent to a compatible almost product structure satisfying $\Pi^{2}=\mathbb{1}$, for $S U(8) / \mathbb{Z}_{2} \subset$ $E_{7(7)}$ the structure is defined by a compatible almost complex structure satisfying $J^{2}=-\mathbb{1}$. Given $J$ and $\Omega$ one can then define the corresponding exceptional generalised metric (EGM) $G$ by

$$
G(X, Y)=\Omega(X, J Y),
$$

which gives a positive definite metric on $E$.

We now turn to how one constructs the generic form of $J$ and hence $G$. Given a metric $\hat{g}_{a b}$ on the $S L(8, \mathbb{R})$ representation space $V$, a natural way to define a particular almost 
complex structure $J_{0}$ (using the conventions of appendix B.1, so that, in particular, pairs of indices $a a^{\prime}$ and $b b^{\prime}$ are antisymmetrised) is as

$$
J_{0} X=\left(\begin{array}{cc}
0 & -\hat{g}^{a b} \hat{g}^{a^{\prime} b^{\prime}} \\
\hat{g}_{a b} \hat{g}_{a^{\prime} b^{\prime}} & 0
\end{array}\right)\left(\begin{array}{l}
x^{b b^{\prime}} \\
x_{b b^{\prime}}^{\prime}
\end{array}\right)=\left(\begin{array}{c}
-\hat{g}^{a b} \hat{g}^{a^{\prime} b^{\prime}} x_{b b^{\prime}}^{\prime} \\
\hat{g}_{a b} \hat{g}_{a^{\prime} b^{\prime}} x^{b b^{\prime}}
\end{array}\right) .
$$

By construction $J_{0}^{2}=-\mathbb{1}$. The corresponding EGM is

$$
G_{0}(X, Y)=\hat{g}_{a b} \hat{g}_{a^{\prime} b^{\prime}} x^{a a^{\prime}} y^{b b^{\prime}}+\hat{g}^{a b} \hat{g}^{a^{\prime} b^{\prime}} x_{a a^{\prime}}^{\prime} y_{b b^{\prime}}^{\prime}
$$

From the definitions $(\overline{\mathrm{B} .3})$ and $(\overline{\mathrm{B} .4})$ of $\Omega$ and $q$ it is clear that $J_{0} \in E_{7(7)}$ provided $\operatorname{det} \hat{g}=1$. Under an infinitesimal $E_{7(7)}$ transformation $\mu \in \mathbf{1 3 3}$ we have

$$
\delta J_{0}=\left[\mu, J_{0}\right]=\left(\begin{array}{cc}
\mu^{+a a^{\prime}}{ }_{b b^{\prime}} & -2 \mu^{+a b} \hat{g}^{a^{\prime} b^{\prime}} \\
-2 \mu_{a b}^{+} \hat{g}_{a^{\prime} b^{\prime}} & -\mu_{a a^{\prime}}^{+} b b^{\prime}
\end{array}\right)
$$

where $\mu_{a b c d}^{ \pm}=\frac{1}{2}\left(\mu_{a b c d} \pm * \mu_{a b c d}\right)$ and $\mu_{a b}^{ \pm}=\frac{1}{2}\left(\mu_{a b} \pm \mu_{b a}\right)$ where indices are raised and lowered using $\hat{g}$. Thus $J_{0}$ is invariant under the subgroup generated by $\mu_{a b}^{-}$and $\mu_{a b c d}^{-}$. As discussed in appendix B.4 this is precisely $S U(8) / \mathbb{Z}_{2}$ (see $(\overline{B .30})$ ).

Given the embedding (2.14) of $G L(7, \mathbb{R}) \subset S L(8, \mathbb{R})$ discussed in detail in appendix B.3, we can define $\hat{g}$ in terms of a seven-dimensional metric $g$ as

$$
\hat{g}_{a b}=(\operatorname{det} g)^{-1 / 4}\left(\begin{array}{cc}
g_{m n} & 0 \\
0 & \operatorname{det} g
\end{array}\right) \text {. }
$$

Acting on elements of $X=x+\omega+\sigma+\tau$ we have

$$
G_{0}(X, X)=2\left(|x|^{2}+|\omega|^{2}+|\sigma|^{2}+|\tau|^{2}\right),
$$

where $|\tau|^{2}=\frac{1}{7 !} \tau_{m . n_{1} \ldots n_{7}} \tau^{m, n_{1} \ldots n_{7}},|\sigma|^{2}=\frac{1}{5 !} \sigma_{n_{1} \ldots n_{5}} \sigma^{n_{1} \ldots n_{5}}$ etc. and, so that the result is a scalar, we have dropped on overall factor of $(\operatorname{det} g)^{1 / 2}$, which is natural, since in writing $X=x+\omega+\sigma+\tau$ we are using the isomorphism (2.16).

Given a seven-dimensional metric $g$ we have been able to write a particular $S U(8) / \mathbb{Z}_{2}$ structure $J_{0}$. A generic structure, given all such structures lie in the same orbit, will be of the form $J=h J_{0} h^{-1}$ where $h \in E_{7(7)}$, or equivalently $G(X, Y)=G_{0}\left(h^{-1} X, h^{-1} Y\right)$. We write $h=\mathrm{e}^{\mu}$ with the Lie algebra element $\mu=\left(\mu_{b}^{a}, \mu_{a b c d}\right) \in 133$. The elements $\mu^{m}{ }_{n}$ generate the $G L(7, \mathbb{R})$ subgroup and acting on $J_{0}$ simply change the form of the metric $g$. The additional components $\mu^{8}{ }_{m}$ and $\mu^{m}{ }_{8}$ modify the form of $\hat{g}(2.35)$. Since only $\mu_{a b}^{+}$acts non-trivially on $J_{0}$, we need only consider transformations with, say, $\mu^{m}{ }_{8}$. Similarly since only $\mu_{a b c d}^{+}$acts non-trivially we can generate a generic $J$ using only, say $\mu_{m n p 8}$. However, $\mu^{m}{ }_{8}$ and $\mu_{m n p 8}$ transformations precisely correspond to the subgroup of $A$ - and $\tilde{A}$-shifts. Thus, given a generic $g$ defining $G_{0}$, the generic EGM can be written as

$$
G(X, Y)=G_{0}\left(\mathrm{e}^{-A-\tilde{A}} X, \mathrm{e}^{-A-\tilde{A}} Y\right) .
$$

This is analogous to the form (2.6) of the generalised metric in generalised geometry. Note also that for non-trivial $A$ and $\tilde{A}, G_{0}$ is an EGM on the untwisted EGT given by $T M \oplus \Lambda^{2} T^{*} M \oplus \Lambda^{5} T^{*} M \oplus\left(T^{*} M \otimes \Lambda^{7} T^{*} M\right)$, while $G$ is an EGM on the twisted bundle $E$ given by (2.26). 


\section{Supersymmetric backgrounds and EGG}

We will now relate the EGG defined in the previous section to eleven-dimensional supergravity and in particular seven-dimensional supersymmetric backgrounds. After identifying the standard decomposition of the supergravity degrees of freedom on such backgrounds, we first briefly review the corresponding connection between type II backgrounds and generalised geometry before turning to EGG.

The physical context we are interested in is where the eleven-dimensional spacetime is topologically a product of a four-dimensional "external" and a seven-dimensional "internal" space

$$
M_{10,1}=M_{3,1} \times M_{7}
$$

If $M_{7}$ is compact we can consider compactifying eleven-dimensional supergravity to give an effective four-dimensional theory. In particular, the effective theory could be supersymmetric. We could also look for particular examples of compactifications which are solutions of the supergravity field equations and preserve some number of supersymmetries. In either case, the geometry of $M_{7}$ is restricted, and some discussion of the latter case appears in [49]. The goal here is to understand how this restricted geometry can be naturally described in terms of EGG structures on $M_{7}$. We will also focus on the low-energy effective theory rather than the on-shell supersymmetric backgrounds.

\subsection{Effective theories and field decompositions}

Given the product (3.1), the tangent bundle decomposes as $T M_{10,1}=T M_{3,1} \oplus T M_{7}$ and all the supergravity fields can be decomposed under a local $\operatorname{Spin}(3,1) \times \operatorname{Spin}(7) \subset \operatorname{Spin}(10,1)$ symmetry. Normally one would derive a four-dimensional effective description by truncating the Kaluza-Klein spectrum of modes on $M_{7}$ to give a four-dimensional theory with a finite number of degrees of freedom. For instance, compactifying on a torus and keeping massless modes, one finds that the degrees of freedom actually arrange themselves into multiplets transforming under $E_{7(7)}$ for the bosons and $S U(8)$ for the fermions.

However, one can also keep the full dependence of all eleven-dimensional fields on both the position on $M_{3,1}$ and $M_{7}$. One can then simply rewrite the eleven-dimensional theory, breaking the local $\operatorname{Spin}(10,1)$ symmetry to $\operatorname{Spin}(3,1) \times \operatorname{Spin}(7)$, so that it is analogous to a four-dimensional theory. This was done explicitly by de Wit and Nicolai [53], retaining all 32 supersymmetries, where it was shown that in general the degrees of freedom fall into $E_{7(7)}$ and $S U(8) / \mathbb{Z}_{2}$ representations. In this paper we will ultimately be interested in such reformulations focusing on only four of the supercharges so that the theory has a structure analogous to $N=1$ four-dimensional supergravity. Note that formally the only requirement for making such rewritings is not that $M_{10,1}$ is topologically a product, but rather that the tangent space $T M_{10,1}$ decomposes into a four- and seven-dimensional part

$$
T M_{10,1}=T \oplus F
$$

For simplicity, here we will concentrate on the case of a product manifold, though all of our analysis actually goes through in the more general case, with the EGT defined in terms of 
$F$ rather than $T M_{7}$. The analogous analysis in terms of generalised geometry for type II compactifications was given in [11, 20].

Let us briefly note how the fields decompose under $\operatorname{Spin}(3,1) \times \operatorname{Spin}(7)$. Our conventions for eleven-dimensional supergravity are summarised in appendix A.1. The degrees of freedom are the metric $g_{M N}$, three-form $A_{M N P}$ and gravitino $\Psi_{M}$. Consider first the $\operatorname{Spin}(3,1)$ scalars. The eleven-dimensional metric decomposes as a warped product

$$
\mathrm{d} s^{2}\left(M_{11}\right)=\mathrm{e}^{2 E} g_{\mu \nu}^{(4)} \mathrm{d} x^{\mu} \mathrm{d} x^{\nu}+g_{m n} \mathrm{~d} x^{m} \mathrm{~d} x^{n},
$$

where $\mu=0,1,2,3$ denote coordinates on the external space. To get a conventionally normalised Einstein term in the four-dimensional effective theory we must take

$$
\mathrm{e}^{-2 E}=\sqrt{\operatorname{det} g}
$$

where det $g$ is the determinant of $g_{m n}$. In a conventional compactification, deformations of the internal metric $g_{m n}$ lead to scalar moduli fields in the effective theory. Moduli fields can also arise from the flux $F$. Keeping only $\operatorname{Spin}(3,1)$ scalar parts, one can decompose

$$
F=*_{7} \tilde{\mathcal{F}} \wedge \mathrm{e}^{4 E} \epsilon_{(4)}+\mathcal{F}
$$

where $\mathcal{F} \in \Lambda^{4} T^{*} M_{7}$ and $\tilde{\mathcal{F}} \in \Lambda^{7} T^{*} M_{7}$ and $\epsilon_{(4)}=\sqrt{-g^{(4)}} \mathrm{d} x^{0} \wedge \cdots \wedge \mathrm{d} x^{3}$. The elevendimensional equation of motion and Bianchi identify (A.4) then decompose as

$$
\begin{array}{rlrl}
\mathrm{d} \tilde{\mathcal{F}}+\frac{1}{2} \mathcal{F} \wedge \mathcal{F} & =0, & \mathrm{~d}\left(\mathrm{e}^{4 E} *_{7} \tilde{\mathcal{F}}\right) & =0, \\
\mathrm{~d} \mathcal{F} & =0, & \mathrm{~d}\left(\mathrm{e}^{4 E} *_{7} \mathcal{F}\right)+\mathrm{e}^{4 E} *_{7} \tilde{\mathcal{F}} \wedge \mathcal{F}=0
\end{array}
$$

so one can introduce, locally,

$$
\begin{aligned}
& \mathcal{F}=\mathrm{d} A, \\
& \tilde{\mathcal{F}}=\mathrm{d} \tilde{A}-\frac{1}{2} A \wedge \mathcal{F},
\end{aligned}
$$

where $A \in \Lambda^{3} T^{*} M_{7}$ and $\tilde{A} \in \Lambda^{6} T^{*} M_{7}$. By definition, $\mathcal{F}$ and $\tilde{\mathcal{F}}$ are globally elements of $\Lambda^{4} T^{*} M$ and $\Lambda^{7} T^{*} M$ respectively. Thus on $U_{(\alpha)} \cap U_{(\beta)}$ we have

$$
\begin{aligned}
& \mathrm{d} A_{(\alpha)}-\mathrm{d} A_{(\beta)}=0 \\
& \mathrm{~d} \tilde{A}_{(\alpha)}-\mathrm{d} \tilde{A}_{(\beta)}=\frac{1}{2}\left(A_{(\alpha)}-A_{(\beta)}\right) \wedge \mathrm{d} A_{(\beta)}
\end{aligned}
$$

This implies that the potentials $A$ and $\tilde{A}$ must patch precisely as given by (2.24). We see that the twisting of the EGT (2.23) is precisely that corresponding to the supergravity potentials. Furthermore, given the discussion of the previous section 2.3 the scalar degrees of freedom $g_{m n}$ and $A_{m n p}$ and $\tilde{A}_{m_{1} \ldots m_{6}}$ scalars can be combined together as an EGM or equivalently an almost complex structure $J$ on $E$.

Turning briefly to the remaining fields, there are 28 bosonic $\operatorname{Spin}(3,1)$ vector degrees of freedom coming from off-diagonal components of the metric $g_{\mu m}$ and from $A_{\mu m n}$. One usually also introduces the corresponding dual potentials giving a total of 56. Finally for the fermionic degrees of freedom we decompose the eleven-dimensional gamma matrices as

$$
\Gamma^{\mu}=\mathrm{e}^{-E} \gamma^{\mu} \otimes \mathbb{1} \quad \Gamma^{m}=\mathrm{i} \gamma_{(4)} \otimes \gamma^{m} .
$$


The seven-dimensional gamma-matrix conventions are defined in A.2, while the fourdimensional gamma matrices are chosen to satisfy $\left\{\gamma_{\mu}, \gamma_{\nu}\right\}=2 g_{\mu \nu}^{(4)} \mathbb{1}$ and $\gamma_{\mu_{1} \ldots \mu_{4}}=\gamma_{(4)} \epsilon_{\mu_{1} \ldots \mu_{4}}^{(4)}$. The real eleven-dimensional spinors correspondingly decompose as

$$
\begin{aligned}
\epsilon & =\mathrm{e}^{E / 2} \theta_{+} \otimes \zeta^{c}+\mathrm{e}^{E / 2} \theta_{-} \otimes \zeta \\
\mathbf{3 2} & =(\mathbf{2}, \mathbf{8})+(\overline{\mathbf{2}}, \overline{\mathbf{8}})
\end{aligned}
$$

where $\mathrm{i} \gamma_{(4)} \theta_{ \pm}= \pm \theta_{ \pm}$(with $\theta_{+}^{c}=D \theta_{+}^{*}=\theta_{-}$and $-\gamma_{\mu}^{*}=D^{-1} \gamma_{\mu} D$ ) are chiral fourdimensional spinors and $\zeta^{c}$ is a complex $\operatorname{Spin}(7)$ spinor. The factor of $\mathrm{e}^{E / 2}$ and the choice of labelling $\zeta$ versus $\zeta^{c}$ are conventional. Thus $\Psi_{\mu}$ decomposes as eight spin- $\frac{3}{2}$ fermions, while $\Psi_{m}$ gives 56 spin- $\frac{1}{2}$ fermions.

As discussed in appendix A.2 there is a natural embedding of $S U(8)$ in the Clifford algebra $\operatorname{Cliff}(7,0 ; \mathbb{R})$ with the complex $\operatorname{Spin}(7)$ spinors transforming in the fundamental representation. In reformulating the eleven-dimensional theory, all the degrees of freedom, fermionic and bosonic arrange as $S U(8)$ representations [53]. Thus we can actually promote the $\operatorname{Spin}(3,1) \times \operatorname{Spin}(7)$ symmetry to $\operatorname{Spin}(3,1) \times S U(8)$. This decomposition corresponds to the $N=8$ four-dimensional supergravity multiplet. It is summarized in table i where $\mathbf{r}_{\mathbf{s}}$ transforms as the $\mathbf{r}$ representation of $S U(8)$ with $\operatorname{Spin}(3,1)$ spin $\mathbf{s}$.

$$
\begin{array}{rlrl}
g_{m n}, A_{m n p}, \tilde{A}_{m_{1} \ldots m_{6}}: & \mathbf{3 5}_{\mathbf{0}}+\overline{\mathbf{3 5}_{\mathbf{0}}} & \Psi_{m}: & \mathbf{5 6}_{\mathbf{1} / \mathbf{2}} \\
g_{\mu m}, A_{\mu m n}+\text { duals }: & \mathbf{2 8}_{\mathbf{1}}+\overline{\mathbf{2 8}} \mathbf{1} & \Psi_{\mu}: \mathbf{8}_{\mathbf{3} / \mathbf{2}} \\
g_{\mu \nu}^{(4)}: & \mathbf{1}_{\mathbf{2}} & &
\end{array}
$$

Table 1: Decomposition of eleven-dimensional supergravity fields under $\operatorname{Spin}(3,1) \times S U(8)$

To summarise, from a EGG perspective, the scalar degrees of freedom define an $S U(8) / \mathbb{Z}_{2}$ structure on the EGT. Given this structure (or rather the existence of a double cover $S U(8)$ structure, which is not always guaranteed) one can then define $S U(8)$ spinors and hence the fermionic degrees of freedom. This is the EGG analogue of requiring a metric, or $O(d)$ structure, and hence a set of vielbeins, before one can define ordinary spinors on a curved manifold.

\subsection{Review of generalized geometry of $N=2$ type II backgrounds}

The generic effective four-dimensional $N=2$ supersymmetric theories arising from type II supergravity compactified on $M_{3,1} \times M_{6}$ were analysed in terms of generalised geometry in [11, 20]. The structure was as follows.

The metric and $B$-field on $M_{6}$ combine into a generalised metric (2.5). This defines a $O(6) \times O(6)$ structure on the generalised tangent space (2.11), that is the decomposition $E=C^{+} \oplus C^{-}$. Assuming the double cover $\operatorname{Spin}(6) \times \operatorname{Spin}(6)$ exists, one can define $\operatorname{Spin}(6)$ spinors on $C^{+}$and $C^{-}$separately. In terms of the original ten-dimensional spinors one has the decomposition

$$
\begin{aligned}
\epsilon^{1} & =\theta_{+}^{1} \otimes \zeta_{-}^{1}+\theta_{-}^{1} \otimes \zeta_{+}^{1} \\
\epsilon^{2} & =\theta_{+}^{2} \otimes \zeta_{ \pm}^{2}+\theta_{-}^{2} \otimes \zeta_{\mp}^{2}
\end{aligned}
$$


where in the second line one takes the upper sign for type IIA and the lower for type IIB. (Here $\zeta_{ \pm}$are complex, chiral Spin(6) spinors, with $\zeta_{-}=\zeta_{+}^{c}$. ) The two spinors $\zeta_{+}^{1}$ and $\zeta_{+}^{2}$ naturally transform under the two spin groups $\operatorname{Spin}(6) \times \operatorname{Spin}(6)$.

Next, we want to concentrate on effective theories with $N=2$ supersymmetry in four dimensions. This means, we want to identify a fixed pair of spinors $\left(\zeta_{+}^{1}, \zeta_{+}^{2}\right)$. The eight four-dimensional supersymmetric parameters are then parametrised by $\left(\theta_{+}^{1}, \theta_{+}^{2}\right)$. In order to be able to use these supersymmetries to decompose all modes of the ten-dimensional fields into $N=2$ multiplets, we must require that $\left(\zeta_{+}^{1}, \zeta_{+}^{2}\right)$ are non-vanishing and globally defined. (We can then project the supersymmetry from an action on $M_{9,1}$ to an action on $M_{3,1}$.) But this condition is the same as requiring each spinor to define an $S U(3)$ structure. Thus we have

$$
N=2 \text { effective theory } \Leftrightarrow S U(3) \times S U(3) \text { structure on } E \text {. }
$$

The structure can be defined by the existence of the generalised metric $G$ on $E$ together with a pair of $\operatorname{Spin}(6) \times \operatorname{Spin}(6)$ spinors $\left(\zeta_{+}^{1}, \zeta_{+}^{2}\right)$.

A conventional $S U(3)$ structure can similarly be defined by a ordinary metric $g$ together with a globally defined, nowhere vanishing spinor $\zeta_{+}$. However, one can also define the structure by a pair of real forms $J \in \Lambda^{2} T^{*} M_{6}$ and $\rho \in \Lambda^{3} T^{*} M_{6}$. In the case where the $S U(3)$ structure is integrable, that is when $M_{6}$ is a Calabi-Yau manifold, $J$ is the Kähler form and $\rho$ is the real part of the holomorphic three-form ${ }^{2} \Omega=\rho+\mathrm{i} \hat{\rho}$. Generically $(J, \rho)$ only define a $S U(3)$ structure if $J \wedge \rho=0$ and $J \wedge J \wedge J=\frac{3}{2} \rho \wedge \hat{\rho}$.

It is natural to ask if the $S U(3) \times S U(3)$ structure can be similarly defined in terms of $O(6,6)$ objects. It can, and the representations in question are the spinors of $\operatorname{Spin}(6,6)$. These are defined as follows. (For more details see appendix A of [20].) For $E_{0}=T M_{6} \oplus$ $T^{*} M_{6}$ the spinor bundle $S\left(E_{0}\right)$ is isomorphic to the bundle of forms

$$
S(E)=\left(\Lambda^{7} T^{*} M_{6}\right)^{-1 / 2} \otimes \Lambda^{*} T^{*} M_{6} .
$$

More generally, for $E$, an extension of the form 2.11), on any patch $U_{(\alpha)}$, a spinor $\Upsilon_{(\alpha)} \in$ $S(E)$ is a sum of forms, with the patching

$$
\Upsilon_{(\alpha)}=\mathrm{e}^{-\mathrm{d} \Lambda_{(\alpha \beta)}} \Upsilon_{(\beta)},
$$

where the action is by wedge product. Spinors of $O(6,6)$ are Majorana-Weyl. The positive and negative helicity spin bundles $S^{ \pm}(E)$ are locally isomorphic to the bundles of even and odd forms $\Lambda^{\text {even/odd }} T^{*} M_{6}$. The Clifford action on $\Upsilon \in S(E)$, viewed as a sum of forms, is given by

$$
(x+\xi) \cdot \Upsilon=i_{x} \Upsilon+\xi \wedge \Upsilon .
$$

The usual spinor bilinear form on $S(E)$ is given by the Mukai pairing $\langle\cdot, \cdot\rangle$ on forms. Explicitly

$$
\left\langle\Upsilon, \Upsilon^{\prime}\right\rangle=\sum_{p}(-)^{[(p+1) / 2]} \Upsilon_{(p)} \wedge \Upsilon_{(6-p)}^{\prime}
$$

\footnotetext{
${ }^{2}$ Note that $\hat{\rho}$ can be determined as a homogeneous function of $\rho$ of degree one.
} 
where the subscripts denote the degree of the component forms in $\Lambda^{*} T^{*} M_{6}$ and $[(p+1) / 2]$ takes the integer part of $(p+1) / 2$. Note that, given the isomorphism (3.13), the ordinary exterior derivative defines a natural generalised Dirac operator d : $S^{ \pm}(E) \rightarrow S^{\mp}(E)$.

Given a generalised metric $G$, on can decompose $\operatorname{Spin}(6,6)$ spinors under $\operatorname{Spin}(6) \times$ $\operatorname{Spin}(6)$. Projecting each subspace $C^{ \pm}$onto $T M$ defines a common Spin(6) subgroup. Under this group $\Upsilon \in S(E)$ transform as a bispinor, that is, as an element of $\operatorname{Cliff}(6,0 ; \mathbb{R})$. Explicitly one can write real $\Upsilon^{ \pm} \in S^{ \pm}$as

$$
\Upsilon^{ \pm}=\zeta_{+} \bar{\zeta}_{ \pm}^{\prime} \pm \zeta_{-} \bar{\zeta}_{\mp}^{\prime}
$$

where $\zeta_{+}, \zeta_{+}^{\prime}$ are ordinary $\operatorname{Spin}(6)$ spinors and elements of the $\operatorname{Spin}(d)$ bundles $S^{+}\left(C^{+}\right)$ and $S^{+}\left(C^{-}\right)$respectively. From this perspective $\Upsilon^{ \pm}$is a matrix. It can be expanded as

$$
\Upsilon^{ \pm}=\sum_{p} \frac{1}{8 p !} \Upsilon_{m_{1} \ldots m_{p}}^{ \pm} \gamma^{m_{1} \ldots m_{p}},
$$

with

$$
\Upsilon_{m_{1} \ldots m_{p}}^{ \pm}=\operatorname{tr}\left(\Upsilon^{ \pm} \gamma_{m_{p} \ldots m_{1}}\right) \in \Lambda^{p} T^{*} M_{6},
$$

and where $\gamma^{m}$ are $\operatorname{Spin}(6)$ gamma-matrices and the trace is over the Spin(6) indices. For $\Upsilon^{+}$only the even forms are non-zero, while for $\Upsilon^{-}$the odd forms are non-zero. This gives an explicit realisation of the isomorphism between $S^{ \pm}(E)$ and $\Lambda^{\text {even/odd }} T^{*} M_{6}$.

One can introduce a pair of $\operatorname{Spin}(6) \times \operatorname{Spin}(6)$ spinors which define the $S U(3) \times S U(3)$ structure. Write the complex objects

$$
\Phi^{+}:=\mathrm{e}^{-B} \zeta_{+}^{1} \bar{\zeta}_{+}^{2}, \quad \Phi^{-}:=\mathrm{e}^{-B} \zeta_{+}^{1} \bar{\zeta}_{-}^{2},
$$

where again $\mathrm{e}^{-B}$ acts by wedge product. Note that in the special case where $\zeta_{+}^{1}=\zeta_{+}^{2}$, the two $S U(3)$ structures are the same, and we have

$$
\Phi^{+}=\frac{1}{8} \mathrm{e}^{-B-\mathrm{i} J}, \quad \Phi^{-}=-\frac{\mathrm{i}}{8} \mathrm{e}^{-B} \Omega .
$$

Generically, each $\Phi^{ \pm}$individually defines an $S U(3,3)$ structure on $E$. Provided these structures are compatible, together they define a common $S U(3) \times S U(3)$ structure. The requirements of compatibility, in terms of the Mukai pairing (3.16) is that [11]

$$
\begin{aligned}
\left\langle\Phi^{+}, V \cdot \Phi^{-}\right\rangle & =\left\langle\bar{\Phi}^{+}, V \cdot \Phi^{-}\right\rangle=0 \quad \forall V \in E, \\
\left\langle\Phi^{+}, \bar{\Phi}^{+}\right\rangle & =\left\langle\Phi^{-}, \bar{\Phi}^{-}\right\rangle .
\end{aligned}
$$

If $\Phi^{ \pm}$are given by (3.20) they are automatically compatible [10]. However, one can also reverse the logic. The $S U(3,3)$ structures can actually be defined using only the real parts $\Upsilon^{ \pm}=\operatorname{Re} \Phi^{ \pm}$. Furthermore any pair of $\operatorname{Spin}(6,6)$ spinors $\left(\Upsilon^{+}, \Upsilon^{-}\right)$satisfying the conditions (3.22) define an $S U(3) \times S U(3)$ structure. So

$$
S U(3) \times S U(3) \text { structure on } E \Leftrightarrow\left(\Upsilon^{+}, \Upsilon^{-}\right) .
$$

The spinor bundles $S^{ \pm}(E)$ are 32-dimensional. The compatibility requirement (3.22) gives 13 conditions. Thus the space of $\left(\Upsilon^{+} \Upsilon^{-}\right)$is 51-dimensional. Different structures 
$\left(\zeta^{+}, \zeta^{-}\right)$can be related by $O(6,6)$ transformations. Since a given structure is $S U(3) \times S U(3)$ invariant, the space of all structures should be related to the coset space $\Sigma=O(6,6) / S U(3) \times S U(3)$, which is 50-dimensional. From this perspective the compatible pair $\left(\Upsilon^{+}, \Upsilon^{-}\right)$give an embedding, as a one-dimensional family of orbits,

$$
\left(\Upsilon^{+}, \Upsilon^{-}\right): \frac{O(6,6)}{S U(3) \times S U(3)} \times \mathbb{R}^{+} \hookrightarrow S^{+}(E) \oplus S^{-}(E)
$$

The additional $\mathbb{R}^{+}$factor corresponds simply to an overall rescaling of the generalised spinors.

The key point of introducing all these structures is that they provide a very simple way to characterise the effective theory. As shown in [11, 20], $\Phi^{ \pm}$parametrise a special Kähler space underlying the vector or hypermultiplet scalar degrees of freedom, and there are simple expressions for the $N=2$ analogues of the superpotential in terms of $\Phi^{ \pm}$.

\section{3 $N=1$ M-theory backgrounds, EGG and $S U(7)$ structures}

We would now like to identify the analogue of the $N=2 S U(3) \times S U(3)$ structure of type II theories for $N=1$ compactifications of eleven-dimensional supergravity. An $S U(3) \times S U(3)$ structure is the generalised geometrical extension of a conventional supersymmetric $S U(3)$ structure and hence, when on-shell, generalises the notion of a CalabiYau three-fold. The structure introduced here is similarly the EGG generalisation of a $G_{2}$ structure on a seven-dimensional manifold.

Identifying an $N=1$ background requires picking out four preferred supersymmetries out of 32 , or equivalently a fixed seven-dimensional spinor $\zeta$ in the general decomposition (3.10). This decomposition is the most general compatible with four-dimensional Lorentz invariance [65], and generically defines a complex $\zeta$ on the internal space. Given an EGM $G$ we have an $S U(8) / \mathbb{Z}_{2}$ structure on $E$, and $\zeta$ transforms as the fundamental representation 8 of double cover $S U(8)$. To define a generic low-energy effective theory the spinor $\zeta$ must be globally defined and nowhere vanishing. The stabilizer group in $S U(8)$ of a fixed element of the vector 8 representation is $S U(7)$. Thus, given a fixed spinor $\zeta$ at each point of $M$, we see that

$$
N=1 \text { effective theory } \Leftrightarrow S U(7) \text { structure on } E \text {. }
$$

The projection $E \rightarrow T M_{7}$ defines a $G L(7, \mathbb{R})$ subgroup of $E_{7(7)}$. Given a EGM $G$, this defines a $\operatorname{Spin}(7) \subset S U(8)$ subgroup. We can then decompose $\zeta$ into real $\operatorname{Spin}(7)$ spinors,

$$
\zeta=\zeta_{1}+\mathrm{i} \zeta_{2}
$$

Each $\zeta_{i}$ is stabilised by a $G_{2} \in \operatorname{Spin}(7)$ subgroup. Thus from the point of the view of the ordinary tangent space $T M_{7}$, if $\zeta_{i}$ are globally defined and non-vanishing we have a pair of $G_{2}$ structures. However, all we really require is a globally defined non-vanishing complex $\zeta$. Thus in general we may not have either $G_{2}$ structure. In analogy to the case where $\zeta$ is real and we have a single $G_{2}$ structure, we can define the complex bilinears

$$
\varphi_{m n p}=\mathrm{i} \bar{\zeta}^{c} \gamma_{m n p} \zeta \quad \text { and } \quad \psi_{m n p q}=\left(*_{7} \varphi\right)_{m n p q}=-\bar{\zeta}^{c} \gamma_{m n p q} \zeta
$$


Locally, the pair of $\zeta_{i}$ are preserved by a $S U(3)$ group.

We have seen that one way to define the structure is as the pair of EGM and $S U(8)$ spinor $(G, \zeta)$. However, as in the type II case, we can also find an element lying in a particular orbit in an $E_{7(7)}$ representation which can also be used to define the structure. We expect that it can be defined as a spinor bilinear. As we discuss in a moment, this space should also correspond to the $N=1$ chiral multiplet space in the four-dimensional effective theory.

Decomposing under $S U(7)$, the $\mathbf{5 6}$ representation has no singlets so cannot have elements stabilized by $S U(7)$. The adjoint 133 does have a singlet. In terms of the spinor $\zeta$, the singlet in $\mu \in \mathbf{1 3 3}$, using its decomposition $\mathbf{1 3 3}=\mathbf{6 3}+\mathbf{3 5}+\overline{\mathbf{3 5}}$ under $S U(8)$, can be written as

$$
\begin{aligned}
\mu_{0} & =\left(\mu_{0}{ }^{\alpha}{ }_{\beta}, \mu_{0 \alpha \beta \gamma \delta}, \bar{\mu}_{0}{ }^{\alpha \beta \gamma \delta}\right), \\
& =\left(\zeta^{\alpha} \bar{\zeta}_{\beta}-\frac{1}{8}(\bar{\zeta} \zeta) \delta^{\alpha}{ }_{\beta}, 0,0\right) .
\end{aligned}
$$

However, it is easy to see that this is stabilized by $U(7)$ rather than $S U(7)$. The next smallest $E_{7(7)}$ representation is 912. (See appendix B.2 for our conventions for $E_{7(7)}$ representations.) We can define the following $S U(7)$-singlet complex element in terms of its $S U(8)$ decomposition, that is $\mathbf{9 1 2}=\mathbf{3 6}+\mathbf{4 2 0}+\overline{\mathbf{3 6}}+\mathbf{4 2} \mathbf{0}$,

$$
\begin{aligned}
\phi_{0} & =\left(\phi_{0}{ }^{\alpha \beta}, \phi_{0}{ }^{\alpha \beta \gamma} \delta, \bar{\phi}_{0 \alpha \beta}, \bar{\phi}_{0 \alpha \beta \gamma}{ }^{\delta}\right) \\
& =\left(\zeta^{\alpha} \zeta^{\beta}, 0,0,0\right),
\end{aligned}
$$

Finally we can form the structure together with the form-field potentials

$$
\phi=\mathrm{e}^{A+\tilde{A}} \phi_{0} .
$$

Such a $\phi$ does indeed define a generic $S U(7)$ structure.

To see this, we first note that under an infinitesimal $E_{7(7)}$ transformation we have

$$
\begin{aligned}
\delta \phi_{0}{ }^{\alpha \beta} & =\left(\mu_{\gamma}^{\alpha} \zeta^{\gamma}\right) \zeta^{\beta}+\zeta^{\alpha}\left(\mu_{\gamma}^{\beta} \zeta^{\gamma}\right), \\
\delta \phi_{0}{ }^{\alpha \beta \gamma}{ }_{\delta} & =0, \\
\delta \bar{\phi}_{0 \alpha \beta} & =0, \\
\delta \bar{\phi}_{0 \alpha \beta \gamma}{ }^{\delta} & =\mu_{\alpha \beta \gamma \epsilon} \zeta^{\epsilon} \zeta^{\delta} .
\end{aligned}
$$

Thus $\phi_{0}$ is stabilized by elements of $E_{7(7)}$ satisfying

$$
\mu_{\beta}^{\alpha} \zeta^{\beta}=0, \quad \mu_{\alpha \beta \gamma \delta} \zeta^{\delta}=0
$$

Since $\bar{\mu}_{\alpha \beta \gamma \delta}=* \mu_{\alpha \beta \gamma \delta}$, the second condition can only be satisfied if $\mu_{\alpha \beta \gamma \delta}=0$. Since $\mu^{\alpha}{ }_{\beta}$ is an element of the adjoint of $S U(8)$ we see that $\phi_{0}$ is indeed stabilised by $S U(7)$. Since $\mathrm{e}^{A+\tilde{A}} \in E_{7(7)}$ the stabilizer of $\phi$ must also be $S U(7)$. Finally, note that the $S U(8)$ representations were defined using the gamma matrices $\hat{\gamma}^{a}$ defined using the seven dimensional metric $g$. Since action by $\mathrm{e}^{A+\tilde{A}}$ generates a generic EGM, we see that (when taken with the choice of generic $g$ and spinor $\zeta$, which are implicit when we write (3.29) ) the action of $\mathrm{e}^{A+\tilde{A}}$ must generate a generic element of the orbit under $E_{7(7)}$. 
Note that we could also define a real object $\lambda=\operatorname{Re} \phi$

$$
\lambda=\mathrm{e}^{A+\tilde{A}}\left(\zeta^{\alpha} \zeta^{\beta}, 0, \bar{\zeta}_{\alpha} \bar{\zeta}_{\beta}, 0\right),
$$

which also manifestly defines the same $S U(7)$ structure. Let $N(E)$ be the $\mathbf{9 1 2}$ representation space based on the EGT $E$, at each point $x \in M_{7}$, we can view $\lambda$ as an embedding of the coset space

$$
\lambda: \frac{E_{7(7)}}{S U(7)} \times \mathbb{R}^{+} \hookrightarrow N(E),
$$

where the $\mathbb{R}^{+}$factor simply corresponds to a rescaling of $\lambda$. We will call this orbit subspace $\Sigma$. There should then be a natural complex structure on $\Sigma$ which allows one to define the holomorphic $\phi$. This is in analogy to $\Upsilon^{ \pm}$and $\Phi^{ \pm}$in generalised geometry. Note that $\lambda$ far from fills out the whole of the $\mathbf{9 1 2}$ representation space. Rather we are considering a very particular orbit. One could always write down the particular non-linear conditions which define the orbit, that is, the analogues of (3.22).

Finally let us also consider how the supergravity fields decompose under the $S U(7)$ subgroup and how these correspond to different $N=1$ multiplets. We have for $S U(7) \subset$ $S U(8)$

$$
\begin{aligned}
8 & =7+1, & & 35=35, \\
28 & =21+7, & 56 & =35+21 .
\end{aligned}
$$

This means we can arrange the degrees of freedom as in table 2. Note that the coset space

\begin{tabular}{lll} 
multiplet & $S U(7)$ rep & fields \\
\hline chiral & $\mathbf{3 5}$ & $g_{m n}, A_{m n p}, \tilde{A}_{m_{1} \ldots m_{7}}, \Psi_{m}$ \\
vector & $\mathbf{2 1}$ & $g_{\mu m}, A_{\mu n p}, \Psi_{m}$ \\
spin- $\frac{3}{2}$ & $\mathbf{7}$ & $g_{\mu m}, A_{\mu n p}, \Psi_{\mu}$ \\
gravity & $\mathbf{1}$ & $g_{\mu \nu}, \Psi_{\mu}$
\end{tabular}

Table 2: Multiplet structure under $S U(7)$

$E_{7(7)} / S U(7)$ actually decomposes into $\mathbf{3 5}+\mathbf{7}+\overline{\mathbf{3 5}}+\overline{\mathbf{7}}$. Thus there are more degrees of freedom in $\lambda$ than chiral degrees of freedom. The same phenomenon appears in the type II case and is associated to the gauge freedom of the extra spin- $\frac{3}{2}$ multiplets. One solution is to assume in a given truncation of the theory that there are no $\mathbf{7}$ degrees of freedom. Note that in this picture we expect there to be a natural Kähler metric on the coset space $\Sigma=E_{7(7)} / S U(7) \times \mathbb{R}^{+}$corresponding to the Kähler metric on the chiral scalar field space of $N=1$ theories [66].

\section{Application: the effective superpotential}

In the previous section we found the objects defining the $S U(7)$ structure relevant to $N=1$ reformulations of eleven-dimensional supergravity. The elements $\phi \in \Sigma$ should correspond to the chiral multiplet degrees of freedom. As such there should be an analogue of the four-dimensional superpotential $W$, as a holomorphic function of $\phi$. In this section, we will 
derive the generic structure of $W$ and show that it can be written in an $E_{7(7)}$ covariant form. This is the analogue of the corresponding generalised geometry calculation in the case of type II given in [11, 20]. Note that the structure of $W$, for the special case of a $G_{2}$ structure was previously derived in 60].

\subsection{Generic form of the effective superpotential}

We will read off $W$ from the variation of the four-dimensional gravitino. Recall that the $N=1$ gravitino variations are given by

$$
\delta \psi_{\mu+}=\nabla_{\mu} \theta_{+}+\frac{1}{2} \mathrm{ie}^{K / 2} W \gamma_{\mu} \theta_{-}+\ldots,
$$

where $W$ is the superpotential and $K$ the Kähler potential. The expressions for $\mathrm{e}^{K / 2} W$ can then be derived directly from the eleven-dimensional gravitino variation (see appendix A.1 for our conventions)

$$
\delta \Psi_{M}=\nabla_{M} \epsilon+\frac{1}{288}\left(\Gamma_{M}^{N P Q R}-8 \delta_{M}^{N} \Gamma^{P Q R}\right) F_{M N P Q}+\ldots,
$$

where the dots denote terms depending on $\Psi_{M}$.

We must first identify the correctly normalised four-dimensional gravitino $\psi_{\mu}$. A naive decomposition $\Psi_{M}=\left(\Psi_{\mu}, \Psi_{m}\right)$ and identifying $\psi_{\mu}$ as part of $\Psi_{\mu}$, leads to cross-terms in the kinetic energy, so instead we first need to diagonalise the four-dimensional gravitino kinetic energy term. This requires the following shift

$$
\tilde{\Psi}_{\mu}:=\Psi_{\mu}+\frac{1}{2} \Gamma_{\mu} \Gamma^{m} \Psi_{m}
$$

One further has to rescale by a factor of $\mathrm{e}^{E}$, and hence identify the four-dimensional gravitino $\psi_{\mu}$ as the $S U(7)$ singlet part

$$
\tilde{\Psi}_{\mu}=\mathrm{e}^{E / 2} \psi_{\mu+} \otimes \zeta^{c}+\mathrm{e}^{E / 2} \psi_{\mu+}^{c} \otimes \zeta+\ldots
$$

where the dots denote non-singlet terms. This rescaling by $\mathrm{e}^{E / 2}$ is the reason for adopting the conventions in the spinor decomposition given in (3.10). Given we can rescale $\zeta^{c}$ by including factors in $\theta_{+}$, we can always choose a normalisation

$$
\bar{\zeta} \zeta=1
$$

This allows us to introduce the projectors

$$
\begin{aligned}
& \Pi_{+}:=\frac{1}{2}\left(1+\mathrm{i} \gamma_{(4)}\right) \otimes \zeta^{c} \bar{\zeta}^{c} \\
& \Pi_{-}:=\frac{1}{2}\left(1-\mathrm{i} \gamma_{(4)}\right) \otimes \zeta \bar{\zeta}
\end{aligned}
$$

such that

$$
\mathrm{e}^{-E / 2} \Pi_{+} \tilde{\Psi}_{\mu}=\psi_{\mu+} \otimes \zeta^{c}
$$

It is now straightforward to calculate $\delta \psi_{\mu}$ in terms of $\zeta^{c}, \mathcal{F}$ and $\tilde{\mathcal{F}}$. By definition

$$
\begin{aligned}
\delta \psi_{\mu} \otimes \zeta^{c} & =\mathrm{e}^{-E / 2} \Pi_{+} \delta \tilde{\Psi}_{\mu}=\mathrm{e}^{-E / 2} \Pi_{+}\left(\delta \Psi_{\mu}+\frac{1}{2} \Gamma_{\mu} \Gamma^{m} \delta \Psi_{m}\right) \\
& =\nabla_{\mu} \theta_{+} \otimes \zeta^{c}+\frac{1}{2} \mathrm{ie}^{K / 2} W \gamma_{\mu} \theta_{-} \otimes \zeta^{c}
\end{aligned}
$$


which gives

$$
\mathrm{e}^{K / 2} W=\frac{1}{4} \mathrm{ie}^{E}\left(4 \bar{\zeta}^{c} \gamma^{m} \nabla_{m} \zeta+\frac{1}{4 !} \mathcal{F}_{m n p q} \bar{\zeta}^{c} \gamma^{m n p q} \zeta-\mathrm{i} *_{7} \tilde{\mathcal{F}} \bar{\zeta}^{c} \zeta\right)
$$

We have used the fact that $\bar{\zeta}^{c} \gamma^{m} \zeta=0$ identically to remove $\nabla_{m} E$ terms. This expression can be put in a more standard form by writing:

$$
\zeta \bar{\zeta}^{c}=\frac{1}{8}\left(\bar{\zeta}^{c} \zeta\right) \mathbb{1}-\frac{1}{8 \cdot 3 !}\left(\bar{\zeta}^{c} \gamma_{m n p} \zeta^{c}\right) \gamma^{m n p}
$$

Thus, since $\bar{\zeta}^{c} \gamma^{m} \zeta=\bar{\zeta}^{c} \gamma^{m n} \zeta=0$, we have

$$
\begin{aligned}
\left(\bar{\zeta}^{c} \gamma^{m} \nabla_{m} \zeta\right)\left(\bar{\zeta}^{c} \zeta\right) & =\bar{\zeta}^{c} \gamma^{m} \nabla_{m}\left(\zeta \bar{\zeta}^{c}\right) \zeta \\
& =\frac{\mathrm{i}}{8 \cdot 3 !} \nabla_{m} \varphi_{n p q}\left(\bar{\zeta}^{c} \gamma^{m} \gamma^{n p q} \zeta\right) \\
& =-\frac{\mathrm{i}}{8 \cdot 4 !}(\mathrm{d} \varphi)_{m n p q}\left(*_{7} \varphi\right)^{m n p q} \\
& =-\frac{\mathrm{i}}{8} *_{7}(\varphi \wedge \mathrm{d} \varphi),
\end{aligned}
$$

where we used (3.27). Similarly,

$$
\frac{1}{4 !} \mathcal{F}_{m n p q} \bar{\zeta}^{c} \gamma^{m n p q} \zeta=-*_{7}(\mathcal{F} \wedge \varphi) .
$$

Hence

$$
\begin{aligned}
{ }_{{ }_{7}} \mathrm{e}^{K / 2} W & =\frac{1}{8} \mathrm{e}^{E}\left[\frac{1}{\bar{\zeta}^{c} \zeta} \varphi \wedge \mathrm{d} \varphi-2 \mathrm{i} \mathcal{F} \wedge \varphi+2 \tilde{\mathcal{F}} \bar{\zeta}^{c} \zeta\right] \\
& =\frac{1}{8} \bar{\zeta}^{c} \zeta \mathrm{e}^{E}[\mathrm{~d}(\tilde{\varphi}-\mathrm{i} A) \wedge(\tilde{\varphi}-\mathrm{i} A)+2 \mathrm{~d} \tilde{A}-\mathrm{id}(A \wedge \tilde{\varphi})]
\end{aligned}
$$

where we have introduced the renormalised $\tilde{\varphi}=\varphi / \bar{\zeta}^{c} \zeta$.

In the case where $\zeta^{c}=\zeta$ we have a global $G_{2}$ structure, our normalisation convention (4.5) implies that $\bar{\zeta}^{c} \zeta=1$ and one finds that 4.13) agrees with that derived in 60. The generic $S U(7)$ case differs form the simple $G_{2}$ case as through the pre-factor $\bar{\zeta}^{c} \zeta$ and the fact that $\tilde{\varphi}$ is no longer real.

\subsection{An $E_{7(7)}$ covariant expression}

In this section we show that one may rewrite the superpotential term (4.13) in a manifestly $E_{7(7)}$ invariant form using the $S U(7)$ structure $\phi \in \mathbf{9 1 2}$. This is the analogue of the $O(6,6)$ invariant expressions for the $N=2$ prepotentials given in [20] for type IIA and IIB theories compactified on $S U(3) \times S U(3)$ structure backgrounds.

We first need to introduce an embedding of the derivative operator into an $E_{7(7)}$ representation. Given the $G L(7)$ decomposition of the EGT given in (2.15), we see that, assuming for the moment we have a metric $g$, we can introduce an operator

$$
D=\left(D^{a b}, D_{a b}\right) \in \mathbf{5 6}
$$

with

$$
D^{m n}=D^{m 8}=D_{m n}=0, \quad D_{m 8}=(\operatorname{det} g)^{1 / 4} \nabla_{m} .
$$


Transforming to spinor indices gives

$$
D=\left(D^{\alpha \beta}, \bar{D}_{\alpha \beta}\right)=\left(-\frac{1}{2 \sqrt{2}} \gamma^{m \alpha \beta} \nabla_{m}, \frac{1}{2 \sqrt{2}} \gamma_{\alpha \beta}^{m} \nabla_{m}\right) .
$$

Given the derivative operator we can define its action on $\phi \in \mathbf{9 1 2}$. In particular using the product between 56 and $\mathbf{9 1 2}$ (see $(\mathrm{B.15})$ or $(\mathrm{B.31})$ ) we can define an object in the $\mathbf{1 3 3}$ representation which we denote as $(D \cdot \phi)^{A B}=D_{C} \phi^{C(A B)}$ (where indices are raised and lowered using the symplectic structure $\left.\Omega_{A B}\right)$.

The claim is that the superpotential can be written as

$$
(D \cdot \phi) \phi=-\left(\frac{3}{4 \sqrt{2}} \mathrm{e}^{-E} \mathrm{e}^{K / 2} W\right) \phi,
$$

where $(D \cdot \phi) \phi \in \mathbf{9 1 2}$ denotes the adjoint action of $D \cdot \phi$ on $\phi$ itself. The statement is that $(D \cdot \phi) \phi$ is itself an $S U(7)$ singlet, proportional to $\phi$ and $W$ is related to the constant of proportionality. Note that we expect $W$ to be a holomorphic function of $\phi$, since $\phi$ encodes the chiral multiplet scalar fields, whereas the Kähler potential should be a function of both $\phi$ and its complex conjugate. This suggests we should identify $\mathrm{e}^{E}$ and $\mathrm{e}^{K / 2}$, or equivalently $\mathrm{e}^{-K}=$ const. $\times \sqrt{\operatorname{det} g}$. We return to this briefly in section 0 .

To show (4.17) requires two steps. First recall that we defined $\phi=\mathrm{e}^{A+\tilde{A}} \phi_{0}$. Writing $h=\mathrm{e}^{A+\tilde{A}} \in E_{7(7)}$, one can then define a new operator $D_{0}$ with a connection taking values in the adjoint of $E_{7(7)}$ by conjugating by $h$. Making the $E_{7(7)}$ indices explicit, we define

$$
D_{0}^{A B}{ }_{C}=D^{A} \delta^{B}{ }_{C}+\kappa^{A B}{ }_{C}
$$

where $\kappa^{A B}{ }_{C}=\left(h^{-1}\right)^{B}{ }_{E} D^{A} h^{E}{ }_{C}$. One then has $D \cdot \phi=\mathrm{e}^{A+\tilde{A}}\left(D_{0} \cdot \phi_{0}\right)$ where

$$
\begin{aligned}
\left(D_{0} \cdot \phi_{0}\right)^{A B} & =\Omega_{C D}\left(D^{C} \phi_{0}^{D(A B)}\right. \\
& +\kappa^{C D}{ }_{E} \phi_{0}^{E(A B)}+\kappa^{C\left(A_{E} \phi_{0}^{D E \mid B)}+\kappa^{C(B}{ }_{E} \phi_{0}^{D \mid A) E}\right),}
\end{aligned}
$$

where we have used the fact that $h^{A}{ }_{B} D^{B}=D^{A}$. The expression (4.17) can thus be rewritten as

$$
\left(D_{0} \cdot \phi_{0}\right) \phi_{0}=-\left(\frac{3}{4 \sqrt{2}} \mathrm{e}^{-E} \mathrm{e}^{K / 2} W\right) \phi_{0}
$$

It is then straightforward to calculate $\left(D_{0} \cdot \phi_{0}\right) \phi_{0}$. Using the Hadamard formula

$$
\mathrm{e}^{P} Q \mathrm{e}^{-P}=Q+[P, Q]+\frac{1}{2}[P,[P, Q]]+\ldots
$$

for operators $P$ and $Q$, one can calculate $D_{0}$. Given the commutator algebra 2.20 , we have

$$
\begin{aligned}
\mathrm{e}^{-A-\tilde{A}} \nabla_{m} \mathrm{e}^{A+\tilde{A}} & =\nabla_{m}+\nabla_{m}(A+\tilde{A})+\frac{1}{2}\left[\nabla_{m}(A+\tilde{A}), A+\tilde{A}\right]+\ldots \\
& =\nabla_{m}+\nabla_{m} A+\nabla_{m} \tilde{A}-\frac{1}{2} \nabla_{m} A \wedge A \\
& :=\nabla_{m}+\kappa_{m}
\end{aligned}
$$


Note that this expression truncates at quadratic order. Again the connection $\kappa_{m}$ takes values in the $E_{7(7)}$ Lie algebra. In the $S U(8) / \mathbb{Z}_{2}$ basis $\phi_{0}$ takes the form (3.29). Hence

$$
\begin{aligned}
\left(\nabla_{m}+\kappa_{m}\right) \phi_{0}{ }^{\alpha \beta} & =\nabla_{m}\left(\zeta^{\alpha} \zeta^{\beta}\right)+\left(\kappa_{m}{ }^{\alpha}{ }_{\gamma}{ }^{\gamma}\right) \zeta^{\beta}+\zeta^{\alpha}\left(\kappa_{m}{ }^{\beta}{ }_{\gamma} \zeta^{\gamma}\right), \\
\left(\nabla_{m}+\kappa_{m}\right) \phi_{0}{ }^{\alpha \beta \gamma}{ }_{\delta} & =0, \\
\left(\nabla_{m}+\kappa_{m}\right) \bar{\phi}_{0 \alpha \beta} & =0, \\
\left(\nabla_{m}+\kappa_{m}\right) \bar{\phi}_{0 \alpha \beta \gamma}{ }^{\delta} & =\kappa_{m \alpha \beta \gamma \epsilon} \zeta^{\epsilon} \zeta^{\delta} .
\end{aligned}
$$

so that

$$
\begin{aligned}
\left(D_{0} \cdot \phi_{0}\right)_{\beta}^{\alpha}=\frac{3 \mathrm{i}}{8 \sqrt{2}}[ & \nabla_{m}\left(\zeta^{\alpha} \zeta^{\gamma}\right) \gamma_{\gamma \beta}^{m} \\
& \left.+\kappa_{m}{ }^{\alpha}{ }_{\gamma} \zeta^{\gamma} \zeta^{\delta} \gamma^{m}{ }_{\delta \beta}-\zeta^{\alpha} \gamma^{m}{ }_{\beta \gamma} \kappa_{m}{ }_{\delta}{ } \zeta^{\delta}-\zeta^{\alpha} \gamma^{m \gamma \delta} \kappa_{m \gamma \delta \beta \epsilon} \zeta^{\epsilon}\right] \\
\left(D_{0} \cdot \phi_{0}\right)_{\alpha \beta \gamma \delta}= & -\frac{\mathrm{i}}{2 \sqrt{2}} \kappa_{m[\alpha \beta \gamma \mid \epsilon} \zeta^{\epsilon} \gamma^{m}{ }_{\delta] \theta} \zeta^{\theta} .
\end{aligned}
$$

Finally, again using the identity $\zeta^{\alpha} \gamma_{\alpha \beta}^{m} \zeta^{\beta}=0$ we find

$$
\begin{aligned}
{\left[\left(D_{0} \cdot \phi_{0}\right) \phi_{0}\right]^{\alpha \beta} } & =-\frac{3 \mathrm{i}}{4 \sqrt{2}}\left(\zeta^{\gamma} \gamma^{m}{ }_{\gamma \delta} \nabla_{m} \zeta^{\delta}+\zeta^{\gamma} \gamma_{\gamma \delta}^{m} \kappa_{m}{ }^{\delta}{ }_{\epsilon} \zeta^{\epsilon}\right) \zeta^{\alpha} \zeta^{\beta} \\
& =-\frac{3 \mathrm{i}}{4 \sqrt{2}}\left[\bar{\zeta}^{c} \gamma^{m}\left(\nabla_{m}+\kappa_{m}\right) \zeta\right] \phi_{0}^{\alpha \beta},
\end{aligned}
$$

with all other components vanishing. We see that, as claimed, $\left(D_{0} \cdot \phi_{0}\right) \phi_{0}$ is proportional to $\phi_{0}$.

Finally we recall that $\kappa_{m}$ corresponded to an " $A$-shift" of $\nabla_{m} A$ and a " $\tilde{A}$-shift" of $\nabla_{m} \tilde{A}-\frac{1}{2} \nabla_{m} A \wedge A$. Only the $S U(8) / \mathbb{Z}_{2}$ adjoint component $\kappa_{m}{ }^{\alpha}{ }_{\beta}$ survives in 4.25 . Using the decomposition (B.30) together with the definitions (B.22) we find this component is given by

$$
\kappa_{m}=\frac{1}{4 \cdot 4 !}\left(\nabla_{m} A\right)_{n p q} \gamma^{n p q}-\frac{1}{4} \mathrm{i}\left[*_{7}\left(\nabla_{m} \tilde{A}-\frac{1}{2} \nabla_{m} A \wedge A\right)\right]_{n} \gamma^{n}
$$

and hence

$$
\begin{aligned}
\bar{\zeta}^{c} \gamma^{m}\left(\nabla_{m}+\kappa_{m}\right) \zeta & =\bar{\zeta}^{c} \gamma^{m} \nabla_{m} \zeta+\frac{1}{4 \cdot 4 !} \mathcal{F}_{m n p q} \bar{\zeta}^{c} \gamma^{m n p q} \zeta-\mathrm{i}\left({ }_{*} \tilde{\mathcal{F}}\right) \bar{\zeta}^{c} \zeta \\
& =-\mathrm{ie}^{-E} \mathrm{e}^{K / 2} W
\end{aligned}
$$

as required.

We now return briefly to a subtlety in the definition of $D$. As written (4.15), $D$ is only defined given a metric $g$. However $\phi$ defines an $S U(7)$ structure on $E$ and hence a metric $g$ (and form fields $A$ and $\tilde{A}$ ). Thus, as written, we can think of $D$ as defined in terms of $\phi$. This is in contrast with the type II $S U(3) \times S U(3)$ case. There the exterior derivative defined a natural generalised Dirac operator d $: S^{ \pm}(E) \rightarrow S^{\mp}(E)$ independent of the structure (or specifically any metric). It is also in contrast with the final result: the final superpotential can be written (4.13) using only the exterior derivative.

The indication is that one can actually define $D$ independently of the metric, such that it has a sensible action on $\phi$. In this sense the differential EGG is set up before introducing 
any structure, as is that case in generalised geometry. One would also expect that such a $D$ is dual to the ECB in the same way that the exterior derivative on $S^{ \pm}(E)$ is dual to the ordinary Courant bracket [⿶]. A subtlety in the generalised geometrical case is that the isomorphism between $S^{ \pm}(E)$ and $\Lambda^{*} T^{*} M$ is not unique: the natural isomorphism is to $\left(\Lambda^{d} T^{*} M\right)^{-1 / 2} \otimes \Lambda^{*} T^{*} M$ [2]. Thus to define the exterior derivative one must rescale by something in $\left(\Lambda^{d} T^{*} M\right)^{-1 / 2}$. We expect something similar in the definitions of $\phi$ and $D$, removing the need for $(\operatorname{det} g)^{1 / 4}$ in 4.15 .

\section{Conclusions}

In this paper we have discussed an extension of generalised geometry applicable to elevendimensional supergravity and for which the symmetry group is the continuous U-duality group $E_{d(d)}$. The general form of such constructions was recently discussed by Hull [49]. Here we specifically focused on generic $N=1$ flux compactifications to four dimensions, for which the relevant symmetry group is $E_{7(7)}$. We showed that $N=1$ supersymmetry implies that there is $S U(7)$ structure on this "exceptional generalised geometry" (EGG) defined by an element $\phi$ in a particular orbit in the 912 representation of $E_{7(7)}$. This is the analogue of the pair of generalised spinors $\Phi^{ \pm}$, each defining a generalised complex structure, which characterise $N=2$ type II backgrounds. In the four-dimensional theory it encodes the chiral multiplet scalar degrees of freedom. As an application we showed that the superpotential for generic $N=1$ flux compactifications could be written as an $E_{7(7)}$-invariant homogeneous, holomorphic function of $\phi$.

In fact, almost all the objects appearing in generalised geometry have analogues in EGG. There is an exceptional generalised tangent space $E$, now combining vectors, twoforms, five-forms and an eight-index tensor. This is twisted by gerbes which capture the topological information encoded in the patching of the three-form supergravity potential $A$ and its dual $\tilde{A}$ - the analogues of the NS-NS $B$-field. There is also a natural exceptional Courant bracket, encoding the differential structure on $E$. The seven-dimensional metric $g$ and potentials $A$ and $\tilde{A}$ then combine to define an $S U(8) / \mathbb{Z}_{2}$ structure on $E$. This is the analogue of the generalised metric, combining metric and $B$-field, that defines an $O(d) \times$ $O(d)$ structure in generalised geometry. If the background has $N=1$ supersymmetry this structure is further refined to $S U(7)$, while for type II six-dimensional $N=2$ backgrounds

\begin{tabular}{|c|c|c|c|}
\hline \multicolumn{2}{|c|}{$\begin{array}{l}\text { generalised geometry } \\
\qquad E_{0}=T M \oplus T M^{*}\end{array}$} & \multicolumn{2}{|c|}{$\begin{array}{c}\text { exceptional generalised geometry } \\
E_{0}=T M \oplus \Lambda^{2} T^{*} M \oplus \Lambda^{5} T^{*} M \oplus\left(T^{*} M \otimes \Lambda^{7} T^{*} M\right)\end{array}$} \\
\hline structure & group & structure & group \\
\hline$\eta$ & $O(d, d)$ & $(\Omega, q)$ & $E_{7(7)}$ \\
\hline$\Pi$ & $G L(d) \times G L(d)$ & $J$ & $G L(28, \mathbb{C})$ \\
\hline$G$ & $O(2 d)$ & $G$ & $O(56)$ \\
\hline$(\eta, \Pi)$ or $(\eta, G)$ & $O(d) \times O(d)$ & $(\Omega, q, J)$ or $(\Omega, q, G)$ & $S U(8) / \mathbb{Z}_{2}$ \\
\hline
\end{tabular}

Table 3: Comparing generalised and exceptional generalised geometry 
the generalised structure is $S U(3) \times S U(3) \subset O(6) \times O(6)$. These parallels are partially summarised in table 3 .

There are a number of obvious extensions of this work one would like to consider. Directly related to the results described here are the questions, first, of the definition of the derivative operator $D$ and, secondly, of the form of the Kähler potential 66]. As discussed in section 4.2, we expect that the definition (4.15) of $D$ can be replaced with one written in terms of the ordinary partial derivative without need for a metric. This should, in an appropriate sense following 狛, be the dual of the exceptional Courant bracket defined in (2.29).

As for the superpotential the Kähler potential (or rather the Kähler metric) can be calculated directly by identifying the four-dimensional kinetic terms in the decomposition of the eleven-dimensional theory. One would again expect the potential to be a $E_{7(7)}$ invariant. We know from the work on type II theories [11, 20] that $\mathrm{e}^{-K}$ is proportional to the metric density $\sqrt{\operatorname{det} g}$. A similarly relation here would be compatible with the expression (4.17) for $W$ being a holomorphic function of $\phi$.

In the type II case we also find [11, 20] that $\mathrm{e}^{-K}$ is proportional to the Hitchin functional [1, 67]. This is true both for the ordinary geometrical case for backgrounds parametrised by an $S U(3)$ structure and for the extension to the generalised geometrical Hitchin functional for $S U(3,3)$ structures $\Phi^{ \pm}$. Hitchin has already introduced a functional for a conventional $G_{2}$ structure. One expects that the $E_{7(7)}$-invariant Kähler potential should be the EGG generalisation of this functional for $S U(7)$ structures defined by $\phi$ which includes the potential $A$ and $\tilde{A}$ degrees of freedom. This also should be the natural generalisation of the action for topological M-theory [68]. As the generalised geometrical functionals necessarily arose at one-loop in the topological B-model [30], one conjecture is that the putative EGG $S U(7)$ functional would appear at one-loop in topological Mtheory. However, note that the extension could also be to the generalised geometrical $G_{2} \times G_{2}$ functional [69].

An obvious question to address is to consider not the four-dimensional effective theory but the on-shell supersymmetric backgrounds [66]. For type II theories, satisfying the sixdimensional Killing spinor equations is equivalent to simple differential conditions on the $S U(3) \times S U(3)$ structures $\left(\Phi^{+}, \Phi^{-}\right)[6,8,10,15]$. One expects a very similar relation in EGG for the $S U(7)$ structure $\phi$ using the operator $D$. These "integrability" conditions on the generalised geometry and EGG structures are the generalisations of the $G$-structure and intrinsic torsion classification of supersymmetric backgrounds [70].

One can also repeat the analysis here for compactifications of eleven-dimensional supergravity to other dimensions, or for that matter for type II theories where the EGG "geometrises" the Ramond-Ramond degrees of freedom [49]. There will also be relations between backgrounds in different dimensions. For instance the four-dimensional type II $N=2$ backgrounds [11, 20] should be encoded in the dimensional reduction of the $N=1$ backgrounds discussed here. In particular, one notes that in terms of the generalised structures $O(6,6) \subset E_{7(7)}$ and for supersymmetry $S U(3) \times S U(3) \subset S U(7)$.

Let us end by noting two further connections. Hull's work [49] was partly motivated by the existence of non-geometrical backgrounds [40]- 48]. The exceptional generalised 
tangent space (2.26) is patched not by generic elements of $E_{7(7)}$ but only elements in the subgroup $G L(7) \ltimes G_{\text {closed }}(M)$, that is the usual geometrical patching of the tangent spaces together with $A$ - and $\tilde{A}$-shifts (by exact forms). It is very natural to extend the twisting to generic $E_{7(7)}$ bundles as discussed in [49]. Such spaces cannot directly describe nongeometrical backgrounds since the underlying space $M$ is still a conventional manifold. Nonetheless they are closely connected to the doubled T-fold and U-fold geometries of 40]. It was argued recently [71] that the generic four-dimensional $N=8$ gauged supergravity theories arise from compactification on a 56-dimensional "megatorus" U-fold. Interestingly such generic supergravities are encoded by an embedding tensor [72] which, like $\phi$, lies in the 912 representation of $E_{7(7)}$. Here, we have focused on $N=1$ theories rather than $N=8$ in four dimensions. The appearance of the $\mathbf{9 1 2}$ representation is nonetheless indirectly connected to the embedding tensor. For $N=8$, the embedding tensor appears (via the $T$-tensor) in the supersymmetry variations of the eight gravitinos and 56 spin- $\frac{1}{2}$ fields. Decomposing under $S U(8)$ the $\mathbf{3 6}$ representation appears in the former and the $\mathbf{4 2 0}$ in the latter. In order to define an $N=1$ theory, we further decompose under $S U(7)$. The $\mathbf{3 6}$ representation decomposes as $\mathbf{3 6}=\mathbf{1}+\mathbf{7}+\mathbf{2 8}$. The first term goes with the $N=1$ gravitino and corresponds to the superpotential term (4.1). In addition the $\mathbf{4 2 0}$ representation decomposes as $\mathbf{4 2 0}=\mathbf{2 2 4}+\mathbf{1 4 0}+\mathbf{3 5}+\mathbf{2 1}$. The chiral multiplets are the $\mathbf{3 5}$ representation (see table 2), and so the $\mathbf{3 5}$ term in the $T$-tensor corresponds the derivative of the superpotential with respect to the chiral scalars. Thus the structure $\phi$ and the $N=8$ embedding tensor share a common 35 representation under the $S U(7)$ decomposition.

\section{Acknowledgments}

We would like to thank Jerome Gauntlett, Jan Louis and, in particular, Mariana Graña and Chris Hull for helpful discussions. P. P. P. thanks the FCT (part of the Portuguese Ministry of Education) for financial support under scholarship SFRH/BD/10889/2002. D. W. is supported by a Royal Society University Research Fellowship and thanks the Aspen Center for Physics, CEA/Saclay and the Isaac Newton Institute for Mathematical Sciences for hospitality during the completion of this work.

\section{A. Conventions}

\section{A.1 Eleven-dimensional supergravity}

We adopt conventions where the eleven-dimensional supergravity action takes the form (see for instance [60])

$$
S=\frac{1}{2 \kappa^{2}} \int_{M_{11}} \sqrt{-g}\left(R-\bar{\Psi}_{M} \Gamma^{M N P} D_{N} \Psi_{P}\right)-\frac{1}{2} F \wedge * F-\frac{1}{6} A \wedge F \wedge F+\ldots
$$

while the variation of the gravitino $\Psi_{M}$ is given by

$$
\delta \Psi_{M}=\nabla_{M} \epsilon+\frac{1}{288}\left(\Gamma_{M}^{N P Q R}-8 \delta_{M}^{N} \Gamma^{P Q R}\right) F_{M N P Q}+\ldots
$$


(The dots represent four-fermi terms and terms coupling $\Psi_{M}$ and $F$.) Here $M, N=$ $0,1, \ldots, 10$ are eleven-dimensional indices, the metric $g$ has signature $(-,+, \ldots,+)$ and $\Gamma_{M}$ are the eleven-dimensional gamma matrices satisfying

$$
\left\{\Gamma_{M}, \Gamma_{N}\right\}=2 g_{M N} \mathbb{1}
$$

with $\Gamma_{M_{1} \ldots M_{11}}=\mathbb{1} \epsilon_{M_{1} \ldots M_{11}}$ where the volume form $\epsilon$ is given by $\epsilon=\sqrt{-g} \mathrm{~d} x^{0} \wedge \cdots \wedge \mathrm{d} x^{10}$. The spinors $\epsilon$ and $\Psi_{M}$ are Majorana. Given the intertwining relation $-\Gamma_{M}^{T}=C^{-1} \Gamma_{M} C$, we define the conjugate spinor $\bar{\Psi}_{M}=\Psi_{M}^{T} C^{-1}$. Note that the equation of motion and Bianchi identity for the flux $F$ read

$$
\mathrm{d} * F+\frac{1}{2} F \wedge F=0, \quad \mathrm{~d} F=0 .
$$

\section{A.2 Cliff $(7,0)$ and seven-dimensional spinors}

Let us also fix our conventions for $\operatorname{Spin}(7)$ (see also Appendix C of [50]). The Clifford algebra $\operatorname{Cliff}(7,0 ; \mathbb{R})$ is generated by the gamma matrices $\gamma_{m}$ with $m=1, \ldots, 7$ satisfying

$$
\left\{\gamma_{m}, \gamma_{n}\right\}=2 g_{m n} \mathbb{1}
$$

One finds $\operatorname{Cliff}(7,0 ; \mathbb{R}) \simeq G L(8, \mathbb{C})$ and hence the spinor representation of the Clifford algebra is complex and eight-dimensional. We define the intertwiners $A$ and $C$ by

$$
\gamma_{M}^{\dagger}=A \gamma_{m} A^{-1}, \quad-\gamma_{m}^{T}=C^{-1} \gamma_{m} C,
$$

with $A^{\dagger}=A, C^{T}=C$ and such that $-\gamma_{m}^{*}=D^{-1} \gamma_{m} D$ with $D=C A^{T}$. Given a spinor $\zeta$ we define the conjugate spinors

$$
\bar{\zeta}^{c}=\zeta^{c \dagger} A, \quad \zeta=D \zeta^{c *} .
$$

Furthermore, we write

$$
\gamma_{m_{1} \ldots m_{7}}=\gamma_{(7)} \epsilon_{m_{1} \ldots m_{7}}
$$

with $\epsilon=\sqrt{g} \mathrm{~d} x^{1} \wedge \cdots \wedge \mathrm{d} x^{7}$. Note that one can choose the gamma matrices such that $\gamma_{(7)}=\mathrm{i}$. The intertwiner $A$ provides an hermitian metric on the spinor space, which is invariant under the subgroup $S U(8) \subset \operatorname{Cliff}(7,0 ; \mathbb{R})$, with a Lie algebra spanned by $\left\{\gamma_{m_{1}, m_{2}}, \gamma_{m_{1} m_{2} m_{3}}, \gamma_{m_{1} \ldots m_{6}}, \gamma_{m_{1} \ldots m_{7}}\right\}$.

The even part of the Clifford algebra generated by the $\gamma_{m n}$ has $\operatorname{Cliff}(7,0 ; \mathbb{R})_{\text {even }} \simeq$ $G L(8, \mathbb{R})$ and hence a real spinor representation with $\zeta=\zeta^{c}$. Thus the spin group $\operatorname{Spin}(7) \subset$ $\operatorname{Cliff}(7,0 ; \mathbb{R})_{\text {even }}$ similarly has a real spinor representation. For real spinors, $\bar{\zeta}=\zeta^{T} C^{-1}$, and $C^{-1}$ provides metric on the spin space. This is invariant under a $\operatorname{Spin}(8)$ group with Lie algebra spanned by $\left\{\gamma_{m_{1} m_{2}}, \gamma_{m_{1} \ldots m_{6}}\right\}$. This can alternatively be described by, for $a, b=1, \ldots 8$

$$
\hat{\gamma}_{a b}=\left\{\begin{array}{cl}
(\operatorname{det} g)^{-1 / 4} \gamma_{m n} & \text { if } a=m, b=n \\
(\operatorname{det} g)^{1 / 4} \gamma_{m} \gamma_{(7)} & \text { if } a=m, b=8 \\
-(\operatorname{det} g)^{1 / 4} \gamma_{n} \gamma_{(7)} & \text { if } a=8, b=n
\end{array}\right.
$$


which generate the $\operatorname{Spin}(8)$ Lie algebra with metric

$$
\hat{g}_{a b}=(\operatorname{det} g)^{-1 / 4}\left(\begin{array}{cc}
g_{m n} & 0 \\
0 & \operatorname{det} g
\end{array}\right) .
$$

Here we have introduced some factors of det $g$ to match the form of $\hat{g}$ used elsewhere in the paper (in particular the decomposition under $G L(7, \mathbb{R})$ given in section B.3). With these conventions, the spinors $\zeta$ are of positive chirality with respect to $\operatorname{Spin}(8)$.

If we make the spinor indices explicit writing $\zeta^{\alpha}$ with $\alpha=1, \ldots, 8$ we can raise and lower spinor indices using the metric $C^{-1}$ so, for instance,

$$
\hat{\gamma}_{a b \alpha \beta}=C_{\alpha \gamma}^{-1} \hat{\gamma}_{a b}{ }^{\gamma}, \quad \hat{\gamma}_{a b}^{\alpha \beta}=\hat{\gamma}_{m n}{ }^{\alpha}{ }_{\gamma} C^{\gamma \beta} \text {. }
$$

One also has the useful completeness relations, reflecting Spin(8) triality,

$$
\begin{gathered}
\hat{\gamma}_{a b}{ }^{\alpha \beta} \hat{\gamma}^{a b}{ }_{\gamma \delta}=16 \delta_{[\gamma}^{[\alpha} \delta_{\delta]}^{\beta]}, \\
\hat{\gamma}_{a b}{ }^{\alpha \beta} \hat{\gamma}^{c d}{ }_{\alpha \beta}=16 \delta_{[a}^{[c} \delta_{b]}^{d]} .
\end{gathered}
$$

\section{B. The exceptional Lie group $E_{7(7)}$}

In this appendix we review some of the properties of the group $E_{7(7)}$ relevant to this paper. A detailed definition of $E_{7(7)}$ and an fairly exhaustive description of its properties can be found in Appendix B of [50] which itself refers to the original work by Cartan [73].

\section{B.1 Definition and the 56 representation}

The group $E_{7(7)}$ can be defined by its action on the basic 56-dimensional representation as follows. Let $W$ be a real 56-dimensional vector space with a symplectic product $\Omega$, then $E_{7(7)}$ is a subgroup of $S p(56, \mathbb{R})$ leaving invariant a particular quartic invariant $q$.

Explicitly one can define $\Omega$ and $q$ using the $S L(8, \mathbb{R}) \subset E_{7(7)}$ subgroup. If $V$ is an eight-dimensional vector space, on which $S L(8, \mathbb{R})$ acts in the fundamental representation, then the $\mathbf{5 6}$ representation decomposes as

$$
56=28+28^{\prime}
$$

the $\mathbf{2 8}$ representation corresponding to $\Lambda^{2} V$ and the $\mathbf{2 8}^{\prime}$ to $\Lambda^{2} V^{*}$. Note that using $\epsilon \in \Lambda^{8} V$, the totally antisymmetric form preserved by the $S L(8, \mathbb{R})$ action on $V$, one can identify $\Lambda^{2} V^{*}$ with $\Lambda^{6} V$. In summary, one identifies

$$
W=\Lambda^{2} V \oplus \Lambda^{2} V^{*}
$$

and writes $X \in W$ as the pair $\left(x^{a b}, x_{a b}^{\prime}\right)$ where $a, b=1, \ldots, 8$.

The symplectic product $\Omega$ is then given by, where $A=1, \ldots, 56$

$$
\Omega(X, Y)=\Omega_{A B} X^{A} Y^{B}=x^{a b} y_{a b}^{\prime}-x_{a b}^{\prime} y^{a b},
$$


and the quartic invariant $q$ is

$$
\begin{aligned}
q(X)= & q_{A B C D} X^{A} X^{B} X^{C} X^{D} \\
= & x^{a b} x_{b c}^{\prime} x^{c d} x_{d a}^{\prime}-\frac{1}{4} x^{a b} x_{a b}^{\prime} x^{c d} x_{c d}^{\prime} \\
& \quad+\frac{1}{96}\left(\epsilon_{a b c d e f g h} x^{a b} x^{c d} x^{e f} x^{g h}+\epsilon^{a b c d e f g h} x_{a b}^{\prime} x_{c d}^{\prime} x_{e f}^{\prime} x_{g h}^{\prime}\right)
\end{aligned}
$$

In what follows it will be useful to use a matrix notation where we write

$$
X^{A}=\left(\begin{array}{l}
x^{a a^{\prime}} \\
x_{a a^{\prime}}^{\prime}
\end{array}\right)
$$

such that the symplectic form

$$
\Omega_{A B}=\left(\begin{array}{cc}
0 & \delta_{a}^{b} \delta_{a^{\prime}}^{b^{\prime}} \\
\delta_{b}^{a} \delta_{b^{\prime}}^{a^{\prime}} & 0
\end{array}\right)
$$

Throughout it is assumed that all pairs of primed and unprimed indices $\left(a, a^{\prime}\right)$ etc are antisymmetrised.

\section{B.2 The 133 and 912 representations}

There are two other representations of interest in this paper. First is the adjoint. By definition it is a 133-dimensional subspace $A$ of the Lie algebra $s p(56, \mathbb{R})$. It decomposes under $S L(8, \mathbb{R})$ as

$$
\begin{aligned}
A & =\left(V \otimes V^{*}\right)_{0} \oplus \Lambda^{4} V^{*} \\
\mu & =\left(\mu_{b}^{a}, \mu_{a b c d}\right) \\
\mathbf{1 3 3} & =\mathbf{6 3}+\mathbf{7 0},
\end{aligned}
$$

where $\left(V \otimes V^{*}\right)_{0}$ denotes traceless matrices, so $\mu^{a}{ }_{a}=0$. The action on the $\mathbf{5 6}$ representation is given by

$$
\begin{aligned}
& \delta x^{a b}=\mu^{a}{ }_{c} x^{c b}+\mu^{b}{ }_{c} x^{a c}+* \mu^{a b c d} x_{c d}^{\prime}, \\
& \delta x_{a b}^{\prime}=-\mu^{c}{ }_{a} x_{c b}^{\prime}-\mu^{c}{ }_{b} x_{a c}^{\prime}+\mu_{a b c d} x^{c d},
\end{aligned}
$$

with $* \mu^{a_{1} \ldots a_{4}}=\frac{1}{4 !} \epsilon^{a_{1} \ldots a_{8}} \mu_{a_{5} \ldots a_{8}}$. In terms of the matrix notation we have $\delta X^{A}=\mu^{A}{ }_{B} X^{B}$ with

$$
\mu_{B}^{A}=\left(\begin{array}{cc}
2 \mu^{a}{ }_{b} \delta_{b^{\prime}}^{a^{\prime}} & * \mu^{a a^{\prime} b b^{\prime}} \\
\mu_{a a^{\prime} b b^{\prime}} & -2 \mu^{b}{ }_{a} \delta_{a^{\prime}}^{b^{\prime}}
\end{array}\right) .
$$

Note that $\mu^{A B}=\mu^{A}{ }_{C} \Omega^{-1 C B}$ is a symmetric matrix. Taking commutators of the adjoint action gives the Lie algebra $\mu^{\prime \prime}=\left[\mu, \mu^{\prime}\right]$

$$
\begin{aligned}
& \mu_{b}^{\prime \prime a}=\left(\mu_{c}^{a} \mu_{b}^{\prime c}-\mu^{\prime a}{ }_{c} \mu_{b}^{c}\right)+\frac{1}{3}\left(* \mu^{a c_{1} c_{2} c_{3}} \mu_{b c_{1} c_{2} c_{3}}^{\prime}-* \mu^{\prime a c_{1} c_{2} c_{3}} \mu_{b c_{1} c_{2} c_{3}}\right), \\
& \mu_{a b c d}^{\prime \prime}=4\left(\mu_{[a}^{e} \mu_{b c d] e}^{\prime}+\mu^{\prime e}{ }_{[a} \mu_{b c d] e}\right) .
\end{aligned}
$$

The other representation of interest in this paper is the $\mathbf{9 1 2}$. The representation space $N$ decomposes under $S L(8, \mathbb{R})$ as

$$
\begin{aligned}
N & =S^{2} V \oplus\left(\Lambda^{3} V \otimes V^{*}\right)_{0} \oplus S^{2} V^{*} \oplus\left(\Lambda^{3} V^{*} \otimes V\right)_{0} \\
\phi & =\left(\phi^{a b}, \phi_{d}^{a b c}, \phi_{a b}^{\prime}, \phi_{a b c}^{\prime}{ }^{d}\right) \\
\mathbf{9 1 2} & =\mathbf{3 6}+\mathbf{4 2 0}+\mathbf{3 6}^{\prime}+\mathbf{4 2 0}^{\prime},
\end{aligned}
$$


where $S^{n} V$ denotes the symmetric product and $\left(\Lambda^{3} V \otimes V^{*}\right)_{0}$ denotes traceless tensors, so $\phi^{a b c}{ }_{c}=0$. The adjoint action of $E_{7(7)}$ on $\phi$ is given by

$$
\begin{aligned}
\delta \phi^{a b} & =\mu^{a}{ }_{c} \phi^{c b}+\mu^{b}{ }_{c} \phi^{a c}-\frac{1}{3}\left(* \mu^{a c d e} \phi_{c d e}^{\prime}{ }^{b}+* \mu^{b c d e} \phi_{c d e}^{\prime}{ }^{a}\right), \\
\delta \phi^{a b c}{ }_{d} & =3 \mu^{[a}{ }_{e} \phi^{b c] e}{ }_{d}-\mu^{e}{ }_{d} \phi^{a b c}{ }_{e}+* \mu^{a b c e} \phi_{e d}^{\prime}+* \mu^{e f[a b} \phi_{e f d}^{\prime}{ }^{c]}-* \mu^{e f g[a} \phi_{e f g}^{\prime}{ }^{b} \delta_{d}^{c]}, \\
\delta \phi_{a b}^{\prime} & =-\mu^{c}{ }_{a} \phi_{c b}^{\prime}-\mu_{b}^{c} \phi_{a c}^{\prime}-\frac{1}{3}\left(\mu_{a c d e} \phi^{c d e}{ }_{b}+\mu_{b c d e} \phi^{c d e}{ }_{a}\right), \\
\delta \phi_{a b c}^{\prime}{ }^{d} & =-3 \mu^{e}{ }_{[a} \phi_{b c] e}^{\prime}{ }^{d}+\mu^{d}{ }_{e} \phi_{a b c}^{\prime}{ }^{e}+\mu_{a b c e} \phi^{e d}+\mu_{e f[a b} \phi^{e f d}{ }_{c]}-\mu_{e f g[a} \phi^{e f g}{ }_{b} \delta_{c]}^{d} .
\end{aligned}
$$

In terms of $S p(56, \mathbb{R})$ indices, we have $\phi^{A B C}$, corresponding to the Young tableau $\frac{A|C|}{B}$ with $\phi^{A B C} \Omega_{A B}=0$. The different components are given by

$$
\begin{aligned}
& \phi^{a a^{\prime} b b^{\prime} c c^{\prime}}=-\frac{1}{12}\left(\epsilon^{a b b^{\prime} c c^{\prime} e f g} \phi_{e f g}^{\prime} a^{a^{\prime}}-\epsilon^{b a a^{\prime} c c^{\prime} e f g} \phi_{e f g}^{\prime}{ }^{b^{\prime}}\right), \\
& \phi^{a a^{\prime} b b^{\prime}}{ }_{c c^{\prime}}=2 \phi^{a b} \delta_{c}^{a^{\prime}} \delta_{c^{\prime}}^{b^{\prime}}-\phi^{a a^{\prime} b}{ }_{c} \delta_{c^{\prime}}^{b^{\prime}}+\phi^{b b^{\prime} a}{ }_{c} \delta_{c^{\prime}}^{a^{\prime}}, \\
& \phi^{a a^{\prime}{ }_{b b^{\prime}}{ }^{\prime} c^{\prime}}=\phi^{a c} \delta_{b}^{a^{\prime}} \delta_{b^{\prime}}^{c^{\prime}}-2 \phi^{a a^{\prime} c}{ }_{b} \delta_{b^{\prime}}^{c^{\prime}}-\phi^{c c^{\prime} a}{ }_{b} \delta_{b^{\prime}}^{a^{\prime}},
\end{aligned}
$$

with $\phi^{a a^{\prime}}{ }_{b b^{\prime}} c c^{\prime}=-\phi_{b b^{\prime}} a a^{\prime} c c^{\prime}$ and identical expressions for $\phi_{a a^{\prime} b b^{\prime} c c^{\prime}}$ etc. but with raised and lowered indices reversed.

Finally we will also need the tensor product

$$
56 \times 912=133+\ldots
$$

In terms of $S p(56, \mathbb{R})$ indices we have $\mu^{A B}=X^{C} \Omega_{C D} \phi^{D(A B)}$, while in terms of $S L(8, \mathbb{R})$ components one finds

$$
\begin{aligned}
\mu_{b}^{a} & =\frac{3}{4}\left(x^{a c} \phi_{c b}^{\prime}-x_{b c}^{\prime} \phi^{c a}\right)+\frac{3}{4}\left(x^{c d} \phi_{c d b}^{\prime}{ }^{a}-x_{c d}^{\prime} \phi_{b}^{c d a}\right), \\
\mu_{a b c d} & =-3\left(\phi_{[a b c}^{\prime}{ }^{e} x_{d] e}^{\prime}+\frac{1}{4 !} \epsilon_{a b c d m_{1} \ldots m_{4}} \phi^{m_{1} m_{2} m_{3}}{ }_{e} x^{m_{4} e}\right) .
\end{aligned}
$$

\section{B.3 A $G L(7)$ subgroup}

As described in section 2.2, the tangent space structure group embeds in the action of $E_{7(7)}$ on the EGT. To make this embedding explicit we must identify a particular $G L(7, \mathbb{R}) \subset$ $S L(8, \mathbb{R}) \subset E_{7(7)}$ subgroup. In this appendix we identify this group and give explicit expressions for part of the $E_{7(7)}$ action in terms of $G L(7, \mathbb{R})$ (that is spacetime tensor) representations.

We start with the embedding of $G L(7, \mathbb{R})$ in $S L(8, \mathbb{R})$ given by the matrix

$$
\left(\begin{array}{cc}
(\operatorname{det} M)^{-1 / 4} M^{m}{ }_{n} & 0 \\
0 & (\operatorname{det} M)^{3 / 4}
\end{array}\right) \in S L(8, \mathbb{R}),
$$

where $M \in G L(7, \mathbb{R})$. If $G L(7, \mathbb{R})$ acts linearly on the seven-dimensional vector space $F$ this corresponds to the decomposition of the eight-dimensional representation space $V$ as

$$
V=\left(\Lambda^{7} F\right)^{-1 / 4} F \oplus\left(\Lambda^{7} F\right)^{3 / 4} .
$$


The 56 representation (B.2) of $E_{7(7)}$ then decomposes as $^{3}$

$$
W=\left(\Lambda^{7} F^{*}\right)^{-1 / 2} \otimes\left[F \oplus \Lambda^{2} F^{*} \oplus \Lambda^{5} F^{*} \oplus F^{*} \otimes\left(\Lambda^{7} F^{*}\right)\right] .
$$

We can write an element of $W$ as

$$
X=x+\omega+\sigma+\tau,
$$

where $x \in\left(\Lambda^{7} F^{*}\right)^{-1 / 2} \otimes F$ etc. If we write the index $m=1, \ldots, 7$ for the fundamental $G L(7, \mathbb{R})$ representation, note that, ignoring the tensor density factor $\left(\Lambda^{7} F^{*}\right)^{-1 / 2}, \tau$ has the index structure $\tau_{m, n_{1} \ldots n_{7}}$, where $n$ labels the $F^{*}$ factor and $n_{1} \ldots n_{7}$ the $\Lambda^{7} F^{*}$ factor. We can make the identification between $G L(7, \mathbb{R})$ indices and $S L(8, \mathbb{R})$ indices explicit by writing (again ignoring the $\left(\Lambda^{7} F^{*}\right)^{-1 / 2}$ factor)

$$
\begin{array}{ll}
x^{m 8}=x^{m} & x^{m n}=\sigma_{1 \ldots 7}^{m n} \\
x_{m 8}^{\prime}=\tau_{m, 1 \ldots 7} & x_{m n}^{\prime}=\omega_{m n},
\end{array}
$$

where $\sigma_{p_{1} \ldots p_{7}}^{m n}=(7 ! / 5 !) \delta_{\left[p_{1}\right.}^{m} \delta_{p_{2}}^{n} \sigma_{\left.p_{3} \ldots p_{7}\right]}$.

We can similarly decompose the $\mathbf{1 3 3}$ representation. We find

$$
\begin{aligned}
A & =\left(V \otimes V^{*}\right)_{0} \oplus \Lambda^{4} V^{*} \\
& =F \otimes F^{*} \oplus \Lambda^{6} F \oplus \Lambda^{6} F^{*} \oplus \Lambda^{3} F \oplus \Lambda^{3} F^{*} .
\end{aligned}
$$

We will be particularly interested in the action of the $\Lambda^{3} F^{*}$ and $\Lambda^{6} F^{*}$ parts of $\mathbf{1 3 3}$ on $X$. Identifying

$$
\begin{aligned}
\mu_{m n p 8} & =\frac{1}{2} A_{m n p} \in \Lambda^{3} V^{*} \\
\mu^{m}{ }_{8} & =-A_{1 \ldots 7}^{\prime m} \in \Lambda^{6} V^{*}
\end{aligned}
$$

where $A_{p_{1} \ldots p_{7}}^{\prime m}=(7 ! / 6 !) \delta_{\left[p_{1}\right.}^{m} \tilde{A}_{\left.p_{2} \ldots p_{7}\right]}$ we have the action in the Lie algebra

$$
(A+\tilde{A}) \cdot X=i_{x} A+\left(i_{x} \tilde{A}+A \wedge \omega\right)+(j A \wedge \sigma-j \tilde{A} \wedge \omega) .
$$

Here we have introduced a new notation. The symbol $j$ denotes the first pure $F^{*}$ index of sections of $F^{*} \otimes\left(\Lambda^{7} F^{*}\right)$. Hence

$$
\left(j \alpha^{(p+1)} \wedge \beta^{(7-p)}\right)_{m, n_{1} \ldots n_{7}}:=\frac{7 !}{p !(7-p) !} \alpha_{m\left[n_{1} \ldots n_{p}\right.} \beta_{\left.n_{p+1} \ldots n_{7}\right]}
$$

\section{B.4 The $S U(8) / \mathbb{Z}_{2}$ subgroup and spinor indices}

The maximal compact subgroup of $E_{7(7)}$ is $S U(8) / \mathbb{Z}_{2}$. In the supersymmetry transformations, the spinors transform in the fundamental representation under (the double cover) $S U(8)$. Thus it is often useful to have the decomposition of the various $E_{7(7)}$ representations in terms of $S U(8) / \mathbb{Z}_{2}$.

In particular, one can use the common Spin(8) subgroup to relate the decompositions under $S L(8, \mathbb{R})$ and $S U(8) / \mathbb{Z}_{2}$. Let $\hat{\gamma}^{a b}$ be $\operatorname{Spin}(8)$ gamma matrices defined in (A.9). We

\footnotetext{
${ }^{3}$ Note that, up to the $\left(\Lambda^{7} F^{*}\right)^{-1 / 2}$ factor, the same embedding of $G L(7, \mathbb{R})$ was identified in $[7]$. This followed from an analysis of $E_{11}$ representations in [57].
} 
can raise and lower $S L(8, \mathbb{R})$ indices using the metric $\hat{g}$. Similarly, spinor indices can be raised a lowered using $C^{-1}$. Under $S U(8) / \mathbb{Z}_{2}$ the $\mathbf{5 6}$ representation decomposes as

$$
\begin{aligned}
X & =\left(x^{\alpha \beta}, \bar{x}_{\alpha \beta}\right) \\
\mathbf{5 6} & =\mathbf{2 8}+\mathbf{2 8} .
\end{aligned}
$$

If the symplectic product takes the form

$$
\Omega(X, Y)=\mathrm{i}\left(x^{\alpha \beta} \bar{y}_{\alpha \beta}-\bar{x}_{\alpha \beta} y^{\alpha \beta}\right)
$$

then $\left(x^{a b}, x_{a b}^{\prime}\right)$ and $\left(x^{\alpha \beta}, \bar{x}_{\alpha \beta}\right)$ are related by

$$
\begin{aligned}
& x^{\alpha \beta}=\frac{1}{4 \sqrt{2}}\left(x^{a b}+\mathrm{i} x^{\prime a b}\right) \hat{\gamma}_{a b}{ }^{\alpha \beta}, \\
& \bar{x}_{\alpha \beta}=\frac{1}{4 \sqrt{2}}\left(x^{a b}-\mathrm{i} x^{\prime a b}\right) \hat{\gamma}_{a b \alpha \beta},
\end{aligned}
$$

with $\hat{\gamma}_{a b}{ }^{\alpha \beta}$ and $\hat{\gamma}_{a b \alpha \beta}$ given by (A.11), or equivalently

$$
\left(\begin{array}{c}
x^{\alpha \beta} \\
\bar{x}_{\alpha \beta}
\end{array}\right)=\frac{1}{4 \sqrt{2}}\left(\begin{array}{cc}
\hat{\gamma}_{a b}{ }^{\alpha \beta} & \mathrm{i} \hat{\gamma}^{a b \alpha \beta} \\
\hat{\gamma}_{a b \alpha \beta} & -\mathrm{i} \hat{\gamma}^{a b}{ }_{\alpha \beta}
\end{array}\right)\left(\begin{array}{c}
x^{a b} \\
x_{a b}^{\prime}
\end{array}\right)
$$

Recall that the $S U(8)$ subgroup of $\operatorname{Cliff}(7,0 ; \mathbb{R})$ leaves the norm $\bar{\zeta} \zeta$ invariant. Since the defining $\mathbf{5 6}$ representation decomposes as a spinor bilinear, both the $\mathbb{1}$ and $-\mathbb{1}$ elements in $S U(8)$ leave $X$ invariant and hence we see explicitly that the subgroup of interest of $E_{7(7)}$ is actually $S U(8) / \mathbb{Z}_{2}$.

Viewing a 56-dimensional index either as a pair of $S L(8, \mathbb{R})$ indices or as a pair of spinor indices, the relation $(\overline{\mathrm{B} .28})$ can be used to convert between $S L(8, \mathbb{R})$ and $S U(8) / \mathbb{Z}_{2}$ decompositions of any other $E_{7(7)}$ representations. In particular, decomposing the adjoint representation $\mu$ under $S U(8) / \mathbb{Z}_{2}$ as $\mathbf{1 3 3}=\mathbf{6 3}+\mathbf{3 5}+\overline{\mathbf{3 5}}$ and writing $\mu=\left(\mu^{\alpha}{ }_{\beta}, \mu^{\alpha \beta \gamma \delta}, \bar{\mu}_{\alpha \beta \gamma \delta}\right)$, with $\bar{\mu}_{\alpha \beta \gamma \delta}=* \mu_{\alpha \beta \gamma \delta}$ and $\mu_{\alpha}^{\alpha}=0$, one finds

$$
\begin{aligned}
& \delta x^{\alpha \beta}=\mu^{\alpha}{ }_{\gamma} x^{\gamma \beta}+\mu^{\beta}{ }_{\gamma} x^{\alpha \gamma}+\mu^{\alpha \beta \gamma \delta} \bar{x}_{\gamma \delta}, \\
& \delta \bar{x}_{\alpha \beta}=-\mu^{\gamma}{ }_{\alpha} \bar{x}_{\gamma \beta}-\mu^{\gamma}{ }_{\beta} \bar{x}_{\alpha \gamma}+\bar{\mu}_{\alpha \beta \gamma \delta} x^{\gamma \delta},
\end{aligned}
$$

with

$$
\begin{aligned}
& \mu_{\beta}^{\alpha}=\frac{1}{4} \mu_{a b}^{-} \hat{\gamma}^{a b \alpha_{\beta}}-\frac{1}{48} \mathrm{i} \mu_{a b c d}^{-} \hat{\gamma}_{\beta}^{a b c d \alpha_{\beta}}, \\
& \mu^{\alpha \beta \gamma \delta}=\frac{1}{16}\left(2 \mu_{a c}^{+} g_{b d}^{(8)}+\mathrm{i} \mu_{a b c d}^{+}\right) \hat{\gamma}^{a b[\alpha \beta} \hat{\gamma}^{c d \gamma \delta]} .
\end{aligned}
$$

where $\mu_{a b}^{ \pm}=\frac{1}{2}\left(\mu_{a b} \pm \mu_{b a}\right)$ and $\mu_{a b c d}^{ \pm}=\frac{1}{2}\left(\mu_{a b c d} \pm * \mu_{a b c d}\right)$, and the anti-symmetrisation in the second line is only over $\alpha, \beta, \gamma$ and $\delta$. Note that in both lines the contraction with the relevant combination of gamma matrices automatically projects onto $\mu_{a b}^{ \pm}$and $\mu_{a b c d}^{ \pm}$, so one could in practice leave out the \pm superscripts.

Finally we can similarly introduce the decomposition of the $\mathbf{9 1 2}$ representation under $S U(8) / \mathbb{Z}_{2}$, writing $\phi=\left(\phi^{\alpha \beta}, \phi^{\alpha \beta \gamma}, \bar{\phi}_{\alpha \beta}, \bar{\phi}_{\alpha \beta \gamma}{ }^{\delta}\right)$. The adjoint action of $E_{7(7)}$ then takes exactly the same form as in (B.12) but with spinor indices replacing $S L(8)$ indices. We can also write the $\mathbf{5 6} \times \mathbf{9 1 2} \rightarrow \mathbf{1 3 3}$ product in terms of spinor indices. One find

$$
\begin{aligned}
\mu_{\beta}^{\alpha} & =\frac{3}{4} \mathrm{i}\left(x^{\alpha \gamma} \bar{\phi}_{\gamma \beta}-\bar{x}_{\beta \gamma} \phi^{\gamma \alpha}\right)+\frac{3}{4} \mathrm{i}\left(x^{\gamma \delta} \bar{\phi}_{\gamma \delta \beta}{ }^{\alpha}-\bar{x}_{\gamma \delta} \phi^{\gamma \delta \alpha}{ }_{\beta}\right), \\
\mu_{\alpha \beta \gamma \delta} & =-3 \mathrm{i}\left(\bar{\phi}_{[\alpha \beta \gamma}{ }^{\epsilon} \bar{x}_{\delta] \epsilon}+\frac{1}{4 !} \epsilon_{\alpha \beta \gamma \delta \mu_{1} \ldots \mu_{4}} \phi^{\mu_{1} \mu_{2} \mu_{3}}{ }_{\epsilon} x^{\mu_{4} \epsilon}\right) .
\end{aligned}
$$


The additional factor of i as compared to the $S L(8)$ expression (B.15) comes from the addition factor of i between the $S L(8)$ and $S U(8) / \mathbb{Z}_{2}$ expressions for the symplectic form (B.3) and (B.26) respectively.

\section{References}

[1] N. Hitchin, "Generalized Calabi-Yau manifolds," Quart. J. Math. Oxford Ser. 54 (2003) 281 [arXiv:math.dg/0209099].

[2] M. Gualtieri, "Generalized Complex Geometry," Oxford University DPhil thesis (2004) [arXiv:math.DG/0401221].

[3] N. Hitchin, "Instantons, Poisson structures and generalized Kähler geometry," Commun. Math. Phys. 265, 131 (2006) [arXiv:math.dg/0503432].

[4] N. Hitchin, "Brackets, forms and invariant functionals," arXiv:math.dg/0508618.

[5] S. Gurrieri, J. Louis, A. Micu and D. Waldram, "Mirror symmetry in generalized Calabi-Yau compactifications," Nucl. Phys. B 654, 61 (2003) [arXiv:hep-th/0211102].

[6] M. Grana, R. Minasian, M. Petrini and A. Tomasiello, "Supersymmetric backgrounds from generalized Calabi-Yau manifolds," JHEP 0408 (2004) 046 [arXiv:hep-th/0406137].

[7] P. Grange and R. Minasian, "Modified pure spinors and mirror symmetry," Nucl. Phys. B 732, 366 (2006) [arXiv:hep-th/0412086].

[8] C. Jeschek and F. Witt, "Generalised $G_{2}$-structures and type IIB superstrings," JHEP 0503 (2005) 053 [arXiv:hep-th/0412280].

[9] P. Berglund and P. Mayr, "Non-perturbative superpotentials in F-theory and string duality," arXiv:hep-th/0504058.

[10] M. Grana, R. Minasian, M. Petrini and A. Tomasiello, "Generalized structures of $N=1$ vacua," JHEP 0511 (2005) 020 [arXiv:hep-th/0505212].

[11] M. Graña, J. Louis and D. Waldram, "Hitchin functionals in $N=2$ supergravity," JHEP 0601 (2006) 008 [arXiv:hep-th/0505264].

[12] P. Koerber, "Stable D-branes, calibrations and generalized Calabi-Yau geometry," JHEP 0508, 099 (2005) [arXiv:hep-th/0506154].

[13] L. Martucci and P. Smyth, "Supersymmetric D-branes and calibrations on general $N=1$ backgrounds," JHEP 0511, 048 (2005) [arXiv:hep-th/0507099].

[14] M. Graña, "Flux compactifications in string theory: A comprehensive review," Phys. Rept. 423, 91 (2006) [arXiv:hep-th/0509003].

[15] C. Jeschek and F. Witt, "Generalised geometries, constrained critical points and Ramond-Ramond fields," arXiv:math/0510131.

[16] I. Benmachiche and T. W. Grimm, "Generalized $N=1$ orientifold compactifications and the Hitchin functionals," Nucl. Phys. B 748, 200 (2006) [arXiv:hep-th/0602241].

[17] J. Louis and S. Vaula, " $N=1$ domain wall solutions of massive type II supergravity as generalized geometries," JHEP 0608, 058 (2006) [arXiv:hep-th/0605063].

[18] R. Minasian, M. Petrini and A. Zaffaroni, "Gravity duals to deformed SYM theories and generalized complex geometry," JHEP 0612, 055 (2006) [arXiv:hep-th/0606257]. 
[19] M. Grana, R. Minasian, M. Petrini and A. Tomasiello, "A scan for new $N=1$ vacua on twisted tori," JHEP 0705 (2007) 031 [arXiv:hep-th/0609124].

[20] M. Grana, J. Louis and D. Waldram, "SU(3) $\times S U(3)$ compactification and mirror duals of magnetic fluxes," arXiv:hep-th/0612237.

[21] A. Tomasiello, "Reformulating Supersymmetry with a Generalized Dolbeault Operator," arXiv:0704.2613 [hep-th].

[22] S. Morris, "Doubled geometry versus generalized geometry," Class. Quant. Grav. 24, 2879 (2007).

[23] P. Koerber and D. Tsimpis, "Supersymmetric sources, integrability and generalized-structure compactifications," JHEP 0708, 082 (2007) [arXiv:0706.1244 [hep-th]].

[24] D. Cassani and A. Bilal, "Effective actions and $N=1$ vacuum conditions from $S U(3) \times S U(3)$ compactifications," JHEP 0709, 076 (2007) [arXiv:0707.3125 [hep-th]].

[25] A. Butti, D. Forcella, L. Martucci, R. Minasian, M. Petrini and A. Zaffaroni, "On the geometry and the moduli space of beta-deformed quiver gauge theories," arXiv:0712.1215 [hep-th].

[26] A. Kapustin, "Topological strings on noncommutative manifolds," Int. J. Geom. Meth. Mod. Phys. 1, 49 (2004) [arXiv:hep-th/0310057].

[27] A. Kapustin and Y. Li, "Topological sigma-models with $H$-flux and twisted generalized complex manifolds," arXiv:hep-th/0407249.

[28] U. Lindstrom, M. Rocek, R. von Unge and M. Zabzine, "Generalized Kähler geometry and manifest $N=(2,2)$ supersymmetric nonlinear sigma-models," JHEP 0507, 067 (2005) [arXiv:hep-th/0411186].

[29] R. Zucchini, "Generalized complex geometry, generalized branes and the Hitchin sigma model," JHEP 0503 (2005) 022 [arXiv:hep-th/0501062].

[30] V. Pestun and E. Witten, "The Hitchin functionals and the topological B-model at one loop," Lett. Math. Phys. 74, 21 (2005) [arXiv:hep-th/0503083].

[31] U. Lindstrom, M. Rocek, R. von Unge and M. Zabzine, "Generalized Kähler manifolds and off-shell supersymmetry," Commun. Math. Phys. 269, 833 (2007) [arXiv:hep-th/0512164].

[32] V. Pestun, "Topological strings in generalized complex space," Adv. Theor. Math. Phys. 11, 399 (2007) [arXiv:hep-th/0603145].

[33] A. Kapustin and E. Witten, "Electric-magnetic duality and the geometric Langlands program," arXiv:hep-th/0604151.

[34] W. Merrell, L. A. P. Zayas and D. Vaman, "Gauged (2,2) Sigma Models and Generalized Kahler Geometry," JHEP 0712, 039 (2007) [arXiv:hep-th/0610116].

[35] A. Kapustin and A. Tomasiello, "The general $(2,2)$ gauged sigma model with three-form flux," JHEP 0711, 053 (2007) [arXiv:hep-th/0610210].

[36] U. Lindstrom, M. Rocek, I. Ryb, R. von Unge and M. Zabzine, "New $N=(2,2)$ vector multiplets," JHEP 0708, 008 (2007) [arXiv:0705.3201 [hep-th]].

[37] N. Halmagyi and A. Tomasiello, "Generalized Kähler Potentials from Supergravity," arXiv:0708.1032 [hep-th]. 
[38] P. Grange and S. Schafer-Nameki, "Towards mirror symmetry à la SYZ for generalized Calabi-Yau manifolds," JHEP 0710, 052 (2007) [arXiv:0708.2392 [hep-th]].

[39] A. Lawrence, T. Sander, M. B. Schulz and B. Wecht, "Torsion and Supersymmetry Breaking," arXiv:0711.4787 [hep-th].

[40] C. M. Hull, "A geometry for non-geometric string backgrounds," JHEP 0510, 065 (2005) [arXiv:hep-th/0406102].

[41] J. Shelton, W. Taylor and B. Wecht, "Nongeometric flux compactifications," JHEP 0510 (2005) 085 [arXiv:hep-th/0508133].

[42] A. Dabholkar and C. Hull, "Generalised T-duality and non-geometric backgrounds," JHEP 0605 (2006) 009 [arXiv:hep-th/0512005].

[43] C. M. Hull, "Global Aspects of T-Duality, Gauged Sigma Models and T-Folds," JHEP 0710 (2007) 057 [arXiv:hep-th/0604178].

[44] C. M. Hull, "Doubled geometry and T-folds," JHEP 0707 (2007) 080 [arXiv:hep-th/0605149].

[45] J. Shelton, W. Taylor and B. Wecht, "Generalized flux vacua," JHEP 0702, 095 (2007) [arXiv:hep-th/0607015].

[46] P. Grange and S. Schafer-Nameki, "T-duality with $H$-flux: Non-commutativity, T-folds and $G \times G$ structure," Nucl. Phys. B 770, 123 (2007) [arXiv:hep-th/0609084].

[47] R. A. Reid-Edwards, "Geometric and non-geometric compactifications of IIB supergravity," arXiv:hep-th/0610263.

[48] C. M. Hull and R. A. Reid-Edwards, "Gauge Symmetry, T-Duality and Doubled Geometry," arXiv:0711.4818 [hep-th].

[49] C. M. Hull, "Generalised geometry for M-theory," arXiv:hep-th/0701203.

[50] E. Cremmer and B. Julia, "The SO(8) Supergravity," Nucl. Phys. B 159 (1979) 141.

[51] B. Julia in Supergravity and Superspace, eds. S. W. Hawking and M. Rocek, CUP, Cambridge (1981);

B. Julia, "Infinite Lie Algebras In Physics," in Unified field theories and beyond : proceedings, 5th Johns Hopkins Workshop on Current Problems in Particle Theory, Johns Hopkins, Baltimore (1981).

[52] C. M. Hull and P. K. Townsend, "Unity of superstring dualities," Nucl. Phys. B 438 (1995) 109 [arXiv:hep-th/9410167].

[53] B. de Wit and H. Nicolai, " $D=11$ Supergravity With Local $S U(8)$ Invariance," Nucl. Phys. B 274, 363 (1986).

[54] H. Nicolai, " $D=11$ Supergravity with Local $S O(16)$ Invariance," Phys. Lett. B 187, 316 (1987).

[55] K. Koepsell, H. Nicolai and H. Samtleben, "An exceptional geometry for $d=11$ supergravity?," Class. Quant. Grav. 17, 3689 (2000) [arXiv:hep-th/0006034].

[56] P. C. West, "E $E_{11}$ and M theory," Class. Quant. Grav. 18, 4443 (2001) [arXiv:hep-th/0104081].

[57] P. C. West, " $E_{11}, S L(32)$ and central charges," Phys. Lett. B 575, 333 (2003) [arXiv:hep-th/0307098]. 
[58] F. Riccioni and P. C. West, "E(11)-extended spacetime and gauged supergravities," JHEP 0802, 039 (2008) [arXiv:0712.1795 [hep-th]].

[59] T. Damour, M. Henneaux and H. Nicolai, "E(10) and a 'small tension expansion' of M theory," Phys. Rev. Lett. 89, 221601 (2002) [arXiv:hep-th/0207267];

A. Kleinschmidt and H. Nicolai, "E(10) and $\mathrm{SO}(9,9)$ invariant supergravity," JHEP 0407, 041 (2004) [arXiv:hep-th/0407101].

[60] T. House and A. Micu, "M-theory compactifications on manifolds with $G_{2}$ structure," Class. Quant. Grav. 22 (2005) 1709 [arXiv:hep-th/0412006].

[61] N. J. Hitchin, "Lectures on special Lagrangian submanifolds," arXiv:math/9907034.

[62] A. Giveon, M. Porrati and E. Rabinovici, "Target space duality in string theory," Phys. Rept. 244, 77 (1994) [arXiv:hep-th/9401139].

[63] C. M. Hull, "Gravitational duality, branes and charges," Nucl. Phys. B 509, 216 (1998) [arXiv:hep-th/9705162].

[64] E. Cremmer, B. Julia, H. Lu and C. N. Pope, "Dualisation of dualities. II: Twisted self-duality of doubled fields and superdualities," Nucl. Phys. B 535 (1998) 242 [arXiv:hep-th/9806106].

[65] A. Lukas and P. M. Saffin, "M-theory compactification, fluxes and $A d S_{4}$," Phys. Rev. D 71 (2005) 046005 [arXiv:hep-th/0403235].

[66] P. Pires Pacheco and D. Waldram, work in progress.

[67] N. J. Hitchin, "The geometry of three-forms in six and seven dimensions," arXiv:math/0010054.

[68] R. Dijkgraaf, S. Gukov, A. Neitzke and C. Vafa, "Topological M-theory as unification of form theories of gravity," Adv. Theor. Math. Phys. 9, 603 (2005) [arXiv:hep-th/0411073], N. Nekrasov, "A la recherche de la M-theorie perdue. Z theory: Casing M/F theory," arXiv:hep-th/0412021,

A. A. Gerasimov and S. L. Shatashvili, "Towards integrability of topological strings. I: Three-forms on Calabi-Yau manifolds," JHEP 0411, 074 (2004) [arXiv:hep-th/0409238].

[69] J. de Boer, P. de Medeiros, S. El-Showk and A. Sinkovics, " $G_{2}$ Hitchin functionals at one loop," arXiv:0706.3119 [hep-th].

[70] J. P. Gauntlett, D. Martelli, S. Pakis and D. Waldram, "G-structures and wrapped NS5-branes," Commun. Math. Phys. 247, 421 (2004) [arXiv:hep-th/0205050] ; J. P. Gauntlett and S. Pakis, "The geometry of $D=11$ Killing spinors," JHEP 0304, 039 (2003) [arXiv:hep-th/0212008].

[71] G. Dall'Agata, N. Prezas, H. Samtleben and M. Trigiante, "Gauged Supergravities from Twisted Doubled Tori and Non-Geometric String Backgrounds," arXiv:0712.1026 [hep-th].

[72] B. de Wit, H. Samtleben and M. Trigiante, "On Lagrangians and gaugings of maximal supergravities," Nucl. Phys. B 655, 93 (2003) [arXiv:hep-th/0212239];

B. de Wit, H. Samtleben and M. Trigiante, "The maximal $D=4$ supergravities," JHEP 0706, 049 (2007) [arXiv:0705.2101 [hep-th]].

[73] E. Cartan "Oeuvres completes T1, Part 1 (These)," Gauthier-Villars Paris 1952 
[74] P. C. West, " $E_{11}$ origin of brane charges and U-duality multiplets," JHEP 0408, 052 (2004) [arXiv:hep-th/0406150];

P. C. West, "Brane dynamics, central charges and $E_{11}$," JHEP 0503, 077 (2005) [arXiv:hep-th/0412336]. 